\title{
Weakly dissipative Faraday waves in 2D large aspect ratio annuli
}

\author{
Victoria Lapuerta, Carlos Martel, José M. Vega* \\ ETSI Aeronáuticos, Universidad Politécnica de Madrid. Plaza Cardenal Cisneros 3, 28040 Madrid. Spain
}

\begin{abstract}
Weakly dissipative parametrically excited (by vertical vibration) surface gravity-capillary waves in a two-dimensional, horizontally periodic container are considered. A set of equations is derived for the coupled evolution of the left- and right-traveling surface waves and the associated mean flow, in the case when the container depth is small compared to its length but large compared to the wavelength of the excited waves. The stability of the spatially uniform standing waves (SWs) is first analyzed and then the large time spatio-temporal behavior of the system beyond threshold is numerically studied. The viscous mean flow is found to drastically affect the dynamics of the system and the resulting surface wave patterns.
\end{abstract}

PACS: 47.20.Ky; 47.20.Ma; 47.35.+i; 47.54.+r

Keywords: Faraday waves: Streaming flow; Gravity-capillary waves; Parametric resonance

\section{Introduction}

Faraday waves $[1,2]$ are excited on the fluid surface upon vertical vibration of the container when the forcing amplitude exceeds a threshold value. These waves show a large variety of complex spatio-temporal behaviors [3-5], especially in large aspect ratio containers [6], and have received a great deal of attention. Unfortunately, several basic issues remain unclear in the weakly nonlinear limit, especially at low viscosity. Among these, no systematic theory is available for the associated mean flow, namely the flow generated by slowly varying (as compared to the vibrating period) forcing terms. Viscosity has a more profound effect [7-11] than that assumed in the usual approaches of the small viscosity limit, where viscous effects only come through a viscous dissipation term added a posteriori to the classical Hamiltonian formulation [3]. Nonzero time-averaged Reynolds stresses in the oscillatory boundary layers attached to the solid walls and the free surface are able to force a significant secondary viscous mean flow in the bulk, outside the boundary layers. The mean flow affects the dynamics of the primary waves at leading order, and thus cannot be ignored. The main object of this paper is to derive a set of equations accounting for this coupled evolution, in a limit that is both realistic and suitable for a not too complex description of the mean 


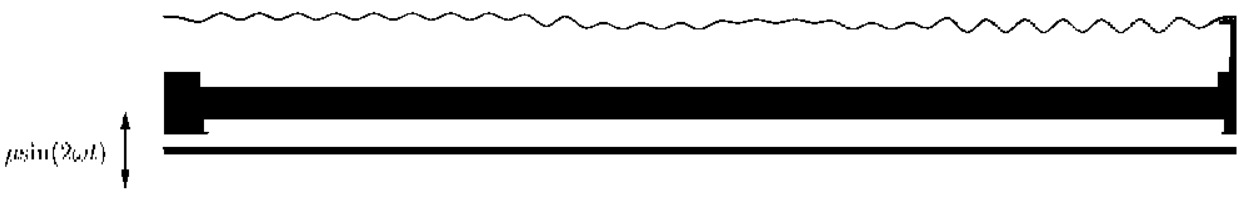

Fig. 1. Sketeh of the fluid domain.

flow. In addition, in order to elucidate the role of the mean flow in the surface wave dynamics, the derived equations will be analyzed by analytical means (when possible) and by direct numerical simulation.

In order to avoid additional difficulties and to clarify the role of the mean flow, we consider the simplest geometry allowing one-dimensional propagation, namely a slender annulus, and for simplicity in the presentation we shall consider a 2D model of that, namely a laterally unbounded layer with periodic boundary conditions (Fig. 1). Results for this geometry could apply even quantitatively to a 3D annular container whose length is large compared to width, and the latter is large compared to the wavelength of the surface waves, provided that width is not too large for the natural frequencies of eigenmodes exhibiting different radial wavenumbers be conveniently separated. In this case, the forcing frequency can be selected such that only one radial mode is excited and propagation is one-dimensional. If, instead width is of the order of wavelength then this latter condition is easily achieved, but the comparison with our theory could only be qualitative due to the effect of the lateral walls.

The remaining of the paper is organized as follows. In the next section we briefly outline the derivation in [8] of a general system of equations for the coupled evolution of the amplitudes of the surface waves and the mean flow. These equations are then simplified in Section 3 for the particular limit case studied in this paper. The simplest nonflat solutions (spatially uniform standing waves (SWs)) and their linear stability are considered in Section 4, and the equations are numerically integrated in Section 5 for representative values of the various parameters to obtain the large time behavior of the system away from threshold. Finally, the main results of the paper are summarized and discussed in Section 6.

\section{Formulation and other preliminaries}

We consider a two-dimensional fluid layer (Fig. 1) above a horizontal plate that is vibrated vertically with an appropriately small amplitude. The layer is laterally unbounded with periodic boundary conditions. We use a Cartesian coordinate system with the $x$-axis along the unperturbed free surface and $y$ vertically upwards, and nondimensionalize space and time with the unperturbed depth $h$ and the gravity-capillary time $\left[g / h+T /\left(\rho h^{3}\right)\right]^{-1 / 2}$, where $g$ is the gravitational acceleration, $\rho$ the density and $T$ the coefficient of surface tension. The nondimensional equations governing the system then are

$$
\psi_{x x}+\psi_{y y}=\Omega, \quad \Omega_{i}-\psi_{y} \Omega_{x}+\psi_{x} \Omega_{y}=C_{z}\left(\Omega_{x x}+\Omega_{y y}\right)
$$

in $-1<y<f$, with boundary conditions

$$
\begin{aligned}
& f_{t}-\psi_{x}-\psi_{y} f_{x}=\left(\psi_{y y}-\psi_{x x}\right)\left(1-f_{x}^{2}\right)-4 f_{x} \psi_{x y}=0, \\
& (1-S) f_{x}-S\left(\frac{f_{x}}{\sqrt{1+f_{x}^{2}}}\right)_{x x}-\psi_{y t}+\psi_{x t} f_{x}-\left(\psi_{x}+\psi_{y} f_{x}\right) \Omega \\
& \quad+\frac{\left(\psi_{x}^{2}+\psi_{y}^{2}\right)_{x}}{2}+\frac{\left(\psi_{x}^{2}+\psi_{y}^{2}\right)_{y} f_{x}}{2}-4 \mu \omega^{2} f_{x} \sin 2 \omega t
\end{aligned}
$$




$$
\begin{aligned}
& =-C_{g}\left[3 \psi_{x x y}+\psi_{y y y}-\left(f_{x x x}+\psi_{x y y}\right) f_{x}\right]+2 C_{g}\left[\frac{2 \psi_{x y} f_{x}^{2}+\left(\psi_{x x}-\psi_{y y}\right) f_{x}}{1+f_{x}^{2}}\right]_{x} \\
& \quad+2 C_{g} \frac{\left(\psi_{x x y}-\psi_{y y y}\right) f_{x}^{2}-\psi_{x y y}\left(1-f_{x}^{2}\right) f_{x}}{1+f_{x}^{2}} \quad \text { at } y=f, \\
& \int_{0}^{L} \Omega_{y} \mathrm{~d} x=\psi=\psi_{y}=0 \quad \text { at } y=-1, \\
& \psi(x+L, y, t) \equiv \psi(x, y, t), \quad f(x+L, t) \equiv f(x, t), \quad \int_{0}^{L} f \mathrm{~d} x=0 .
\end{aligned}
$$

Here $\psi$ is the streamfunction, such that the velocity $(u, v)=\left(-\psi_{y}, \psi_{x}\right), \Omega$ is the vorticity, and $f$ is the free surface elevation required to satisfy volume conservation recalled in the third condition of (4). First condition of (3) is necessary in order that the pressure be periodic in $x$. The remaining equations and boundary conditions are standard. The resulting problem depends on the aspect ratio $L$, on the nondimensional vibration amplitude $\mu$ and frequency $2 \omega$, on the capillary -gravity number $C_{g}=v /\left\lfloor g / h^{3}+T h / \rho\right\rfloor^{1 / 2}$, where $v$ is the kinematic viscosity, and on the gravity-capillary balance parameter $S=T /\left(T+\rho g h^{2}\right)$. Note that $C_{\mathrm{g}}$ and $S$ are related to the usual Onhesorge number $C=v[\rho /(T h)]^{1 / 2}$ and the Bond number $B=\rho g h^{2} / T$ by $C_{g}=C /(1+B)^{1 / 2}$ and $S=1 /(1+B)$. Thus $0 \leq S \leq 1$, and the cxtreme values, $S=0$ and 1 , correspond to the purcly gravitational $(T=0)$ and the purely capillary $(g=0)$ limits, respeclively.

As a starling point to derive the relevant equations governing the coupled cvolution of the surface waves and the mean flow, we shall use a set of general coupled amplitude-mean flow (GCAMF) equations, first oblained in [8], which apply under the assumptions: (a) viscosity is small, (b) the aspect ratio is large, (c) the evolution is weakly nonlinear, which requires that the wave steepness be small, and (d) the relevant Fourier modes in the horizontal coordinate are concentrated around a discrete set of values of the wave number. These four assumptions are collected here for convenience

$$
\left(1+k_{0}\right)\left(\frac{C_{g}}{\omega}\right)^{1 / 2} \ll 1, \quad L \gg 1, \quad\left|\psi_{x}\right|+\left|\psi_{y}\right| \ll 1, \quad|f| \ll 1 .
$$

The spatial Fourier transforms of $\psi$ and $f$ peak for all time around the wavenumbers

$$
\pm m k_{0}, \text { with } m=0,1, \ldots,
$$

and require in particular that $C_{g} \ll 1$. Here $k_{0}$ denotes the wavenumber of the parametrically excited surface mode, defined in terms of the forcing frequency $2 \omega$ as

$$
\omega=\omega\left(k_{0}\right)
$$

where $\omega(k)$ is the irviscid dispersion relation of the surface waves

$$
\omega(k)=\left\lceil\left(1-S+S k^{2}\right) k \tanh k\right\rceil^{1 / 2},
$$

whose associated eigenfunctions are proportional to $(\psi, f)=\left(\Psi_{0}, 1\right)$, with

$$
\Psi_{0}=\frac{\omega \sinh [k(y+1)]}{k \sinh k} .
$$

For convenience, below we shall always use the basic wavenumber $k$ defined as

$$
k=\frac{2 \pi N}{L},
$$


where $N \gg 1$ is an integer such that

$$
-\pi<k_{0} L-2 \pi N \leq \pi \text {. }
$$

This selection shifts the wavenumber $k_{0}$ to the nearest wavenumber commensurate with the imposed spatial periodicity and leads to periodic boundary conditions for the amplitude equations. Note that

$$
\left|k-k_{0}\right| \sim L^{-1} \ll 1
$$

Now, according to assumptions (5) and (6), the streamfunction (and vorticity) in the bulk and the free surface clevation can be decomposed into three parts, namely (i) two counter-propagating wavetrains associated with the surface modes that are parametrically excited; these are slowly modulated both in space and time around a basic frequency $\omega$ and wavenumbers $\pm k$; (ii) a mean flow, which depends weakly on time but can exhibit significant dependence on the space variables $x$ and $y$; and (iii) the remaining part of the solution, which will be called nonresonant. Thus, the solution in the bulk can be written as

$$
\begin{aligned}
& f=\mathrm{e}^{\mathrm{i} k t t}\left(A^{+} \mathrm{e}^{\mathrm{i} k x}+A^{-} \mathrm{e}^{-\mathrm{i} k x x}\right)+\gamma_{1} A^{+} \bar{A}^{-} \mathrm{e}^{2 \mathrm{i} k x}+\gamma_{2} \mathrm{e}^{2 \mathrm{i} / \omega t}\left[\left(A^{+}\right)^{2} \mathrm{e}^{2 \mathrm{i} k x}+\left(A^{-}\right)^{2} \mathrm{e}^{-2 \mathrm{i} k x}\right] \\
& + \text { c.c. }+ \text { HOT }+f^{m}+\text { NRT, } \\
& \psi=\Psi_{0} \mathrm{e}^{\mathrm{i} \omega t}\left(\Lambda^{+} \mathrm{e}^{\mathrm{i} k x}-\Lambda^{-} \mathrm{e}^{-\mathrm{i} k x}\right)+\gamma_{3} \Psi_{22} \mathrm{e}^{2 \mathrm{i} \omega t}\left[\left(\Lambda^{+}\right)^{2} \mathrm{e}^{2 \mathrm{i} k x}-\left(\Lambda^{-}\right)^{2} \mathrm{e}^{-2 \mathrm{i} k x}\right]+\text { c.c. }+ \text { HOT }+\psi^{m}+\mathrm{NRT}, \\
& \Omega=\mathrm{i} \omega^{-1} \mathrm{c}^{\mathrm{i} \omega t t}\left[\left(A^{+} \mathrm{c}^{\mathrm{i} k x}-A^{-} \mathrm{c}^{-\mathrm{i} k x x}\right) \Psi_{0}^{\prime} \Omega_{x}^{m}-\mathrm{i} k\left(A^{+} \mathrm{c} i k x+A^{-} \mathrm{c}^{-\mathrm{i} k x}\right) \Psi_{0} \Omega_{y}^{m}\right]+\text { c.c. }+ \text { HOT }+\Omega^{m}+\text { NRT }
\end{aligned}
$$

Here the superscript $m$ denotes the mean flow variables, and NRT and HOT stand for nonresonant terms and higher order terms, respectively. $\Psi_{0}$ is as defined above, in (9), and the complex amplitudes $A^{ \pm}$depend weakly on $t$ and $x$, while $f^{m}, \psi^{m}$ and $\Omega^{m}$ depend weakly on $\iota$ but strongly on $x$ (and $y$ ). i.e.

$$
\left|A_{x}^{ \pm}\right|+\left|A_{t}^{ \pm}\right| \ll\left|A^{ \pm}\right| \ll 1, \quad\left|f_{t}^{m}\right| \ll\left|f^{m}\right| \ll 1, \quad\left|\psi_{i}^{m}\right| \ll\left|\psi^{m}\right| \ll 1, \quad\left|\Omega_{t}^{m}\right| \ll\left|\Omega^{m}\right| \ll 1 .
$$

The coefficients $\gamma_{1}, \gamma_{2}, \gamma_{3}$ and the function $\Psi_{22}$ in (13) and (14) are given by

$$
\begin{aligned}
& \gamma_{1}=\frac{\left(\sigma^{2}+1\right) \omega^{2}}{\sigma^{2}\left(1-S+4 S k^{2}\right)}, \quad \gamma_{2}=\frac{\left(3-\sigma^{2}\right) k\left(1-S+S k^{2}\right)}{2 \sigma\left\lceil(1-S) \sigma^{2}-S k^{2}\left(3-\sigma^{2}\right)\right]} \\
& \gamma_{3}=\frac{3 \omega\left\lfloor(1-S)\left(1-\sigma^{2}\right)+S k^{2}\left(3-\sigma^{2}\right)\right]}{2 \sigma\left[(1-S) \sigma^{2}-S k^{2}\left(3-\sigma^{2}\right)\right]}, \quad \Psi_{22}=\frac{\sinh [2 k(y+1)]}{\sinh (2 k)},
\end{aligned}
$$

where $\sigma=\tanh k$, as obtained by solving two nonresonant problems at order $\left|\Lambda^{ \pm}\right|^{2}$. Note that $\gamma_{2}$ and $\gamma_{3}$ diverge at $(1-S) \sigma^{2}=s k^{2}\left(3-\sigma^{2}\right)$, i.e., when the strictly inviscid eigenfrequency (8) satisfies $\omega(2 k)=2 \omega(k)$. In the present paper we do not pursue this $2: 1$ resonance further; see $[12,13]$ for a strictly inviscid analysis, and [14-16] for nearly inviscid descriptions that ignore the mean llow.

The complex amplitudes $A^{ \pm}$and the mean flow variables $\psi^{m}, \Omega^{m}$, and $f^{m}$ cvolve (in a timescale that is large compared to the basic period of the waves $2 \pi / \omega)$ according to some cquations that are obtained as solvability conditions for the equations giving the resonant terms at higher order. This requires, as a previous step, to analy $/ \mathrm{e}$ the oscillatory boundary layers attached to the supporting plate and the free surface, to obtain the appropriate boundary conditions for the solution outside these layers, where the expansions (13)-(15) apply. All these have becn done in [8] to obtain the following cvolution equations, called GCAMF cquations, which apply under the sole 
assumptions (5) and (6). To the approximation relevant here, the complex amplitudes are given by

$$
\begin{aligned}
& \Lambda_{t}^{ \pm} \mp v_{\mathrm{g}} \Lambda_{x}^{ \pm}= \mathrm{i} \alpha \Lambda_{x x}^{ \pm}-(\delta+\mathrm{i} d) \Lambda^{ \pm}+\mathrm{i}\left(\alpha_{3}\left|\Lambda^{ \pm}\right|^{2}-\alpha_{4}\left|\Lambda^{\mp}\right|^{2}\right) \Lambda^{ \pm}+\alpha_{5} \mu \bar{\Lambda}^{\mp} \\
& \pm \mathrm{i} \alpha_{6} \int_{-1}^{0} g(y)\left\langle\psi_{y}^{m}\right\rangle^{x} \mathrm{~d} y A^{ \pm}+\mathrm{i} \alpha_{7}\left\langle f^{m}\right)^{x} A^{ \pm} \\
& \Lambda^{ \pm}(x+L, t) \equiv \Lambda^{ \pm}(x, t),
\end{aligned}
$$

and the mean flow is given by

$$
\psi_{x x}^{m}+\psi_{y y}^{n}=\Omega^{m}, \quad \Omega_{t}^{m}-\left[\psi_{y}^{m}+\left(\left|\Lambda^{+}\right|^{2}-\left|\Lambda^{-}\right|^{2}\right) g(y)\right] \Omega_{x}^{n}+\psi_{x}^{m} \Omega_{y}^{m}=C_{g}\left(\Omega_{x x}^{m}+\Omega_{y y}^{m}\right)
$$

in $-1<y<0$, with boundary conditions

$$
\begin{aligned}
& \psi_{x}^{m}-f_{t}^{m}=\beta_{1}\left(\left|\Lambda^{-}\right|^{2}-\left|\Lambda^{+}\right|^{2}\right)_{x}, \quad \psi_{y y}^{m}=\beta_{2}\left(\left|\Lambda^{+}\right|^{2}-\left|\Lambda^{-}\right|^{2}\right), \\
& (1-S) f_{x}^{m}-S f_{x x x}^{m}-\psi_{y t}^{m}+C_{g}\left(\psi_{y y y}^{m}+3 \psi_{x x y}^{m}\right)=-\beta_{3}\left(\left|\Lambda^{+}\right|^{2}+\left|\Lambda^{-}\right|^{2}\right)_{x} \quad \text { at } y=0, \\
& \int_{0}^{L} \Omega_{y}^{m} \mathrm{~d} x=\psi^{m}=0, \quad \psi_{y}^{m}=-\beta_{4}\left[\mathrm{i} \Lambda^{+} \bar{\Lambda}^{-} \mathrm{e}^{2 \mathrm{i} k x}+\text { c.c. }+\left|\Lambda^{-}\right|^{2}-\left|\Lambda^{+}\right|^{2}\right\rceil \quad \text { at } y=-1, \\
& \psi^{m}(x+L, y, t) \equiv \psi^{m}(x, y, l), \quad f^{m}(x+L, l) \equiv f^{m}(x, l), \\
& \int_{0}^{L} f^{m}(x, t) \mathrm{d} x=0 .
\end{aligned}
$$

The horizontal mean value $\langle\cdot\rangle$ is defined as

$$
\langle G(x, y, t)\rangle^{x}=(2 \ell)^{-1} \int_{x-\ell^{\ell}}^{x+\ell} G(z, y, t) \mathrm{d} z, \text { with } 1 \ll \ell \ll L,
$$

$\delta>0$ and $d$ are the (lincar) damping ratio and the effective detuning, respectively, and are given by

$$
\delta=\alpha_{1} C_{g}^{1 / 2}+\alpha_{2} C_{g}, \quad d=\alpha_{1} C_{g}^{1 / 2}-\left(\frac{2 \pi N}{L}-k_{0}\right) v_{\mathrm{g}}
$$

where $^{1}$

$$
\alpha_{1}=\frac{k(\omega / 2)^{1 / 2}}{\sinh (2 k)}, \quad \alpha_{2}=\left[2+\frac{1+\tanh ^{2} k}{4 \sinh ^{2} k}\right] k^{2} .
$$

The group velocity $v_{\mathrm{g}}$ and the dispersion $\alpha$ are calculated from the dispersion relation (8) as

$$
v_{g}=\omega^{\prime}(k), \quad \alpha=-\frac{\omega^{\prime \prime}(k)}{2} .
$$

The remaining coefficients in (18), $\alpha_{3}, \ldots, \alpha_{7}$, and the function $g$ are

$$
\begin{aligned}
& \alpha_{3}=\frac{\omega k^{2}\left[(1-S)\left(9-\sigma^{2}\right)\left(1-\sigma^{2}\right)+S k^{2}\left(7-\sigma^{2}\right)\left(3-\sigma^{2}\right)\right]}{4 \sigma^{2}\left[(1-S) \sigma^{2}-S k^{2}\left(3-\sigma^{2}\right)\right]}+\frac{\left[8(1-S)+5 S k^{2}\right] \omega k^{2}}{4\left(1-S+S k^{2}\right)}, \\
& \alpha_{4}=\frac{\omega k^{2}}{2}\left[\frac{\left(1-S+S k^{2}\right)\left(1+\sigma^{2}\right)^{2}}{\left(1-S+4 S k^{2}\right) \sigma^{2}}+\frac{4(1-S)+7 S k^{2}}{1-S+S k^{2}}\right],
\end{aligned}
$$

1 'These coefficients are taken from [17], except for an crror in the expression of $\alpha_{2}$ (cf. [18]); we are indebted to Flena Martin for pointing out this to us. 


$$
\begin{aligned}
& \alpha_{5}=\omega k \sigma, \quad \alpha_{6}=\frac{k \sigma}{2 \omega}, \quad \alpha_{7}=\frac{\omega k\left(1-\sigma^{2}\right)}{2 \sigma}, \\
& g(y)=\frac{2 \omega k \cosh [2 k(y+1)]}{\sinh ^{2} k} .
\end{aligned}
$$

And the cocfficients $\beta_{1}, \ldots, \beta_{4}$ in (21) and (22) are

$$
\beta_{1}=\frac{2 \omega}{\sigma}, \quad \beta_{2}=\frac{8 \omega k^{2}}{\sigma}, \quad \beta_{3}=\frac{\left(1-\sigma^{2}\right) \omega^{2}}{\sigma^{2}}, \quad \beta_{4}=\frac{3\left(1-\sigma^{2}\right) \omega k}{\sigma^{2}} .
$$

Note that

$$
\alpha_{6} \int_{-1}^{0} g(y) \mathrm{d} y=k
$$

as required from invariance of (18)-(21) under the action $x \rightarrow x+c t, \psi \rightarrow \psi+c y, A^{ \pm} \rightarrow A^{ \pm} \mathrm{e}^{\text {Fikc }}$, which results from Galilean invariance of Eqs. (1) and (2) in the original problem.

The GCAMF equations (18)-(24) are valid in the general limit (5) and (6), which allows several sub-limits depending on the relative values of $C_{g}, L$ and $k$. For simplicity, we assume that the forcing amplitude is such that the forcing and damping terms are of the same order in (18). In this case, the dissipation time, $\delta^{-1}$, is the characteristic time for the slow evolution of the complex amplitudes. The limit considered in [8] was

$$
\delta \ll \frac{v_{\mathrm{g}}}{L},
$$

in which the dissipation time is large compared to the residence time of the surface waves when they travel with the group velocity, $t_{\mathrm{R}}=L / v_{\mathrm{g}}$. In this limit, the wave envelopes just travel undeformed with the group velocity in the timescale $t_{\mathrm{R}}$; and in the longer dissipation timescale each counter-propagating wave only "sees" a spatial average of the other wave and of the mean flow, and thus its evolution is coupled only through these spatial averages and the resulting equations are nonlocal. Also, the coupling between the surface waves and the mean flow is somewhat weak [8], and the complex amplitudes of the surface waves can be decoupled (upon a change of variables) from the mean flow; see [19] for the analysis of these decoupled equations. This decoupling is fortunate because the mean flow equations include a Navier-Stokes-like equation whose study by analytical means is quite limited. Moreover, the mean flow exhibits a $\mathrm{O}(1)$-wavelength in the $x$-direction, which makes numerical calculations quite costly $[10]$. This is due to the forcing terms resulting from the oscillatory boundary layer attached to the lower plate, and could be avoided by considering the limit $k \gg 1$, but then the mean flow becomes unforced and dies out for large time.

Here we consider the case $\delta \sim v_{\mathrm{g}} / L$ and, in order to avoid that the streaming flow exhibits a $\mathrm{O}(1)$-wavelength in the $x$-direction, we assume that the wavenumber of the surface waves is somewhat large. Since the lower boundary layer (which was responsible for the forcing term exhibiting a $\mathrm{O}(1)$ wavelength, see the third condition of (22)) produces a forcing term that decays exponentially as $k \rightarrow \infty$, this can be neglected and the mean flow is forced only at the boundary layer attached to the free surface. But in order to avoid convective terms in the streaming flow momentum equation (see below), we do not allow $k$ being too large. In fact, for simplicity we consider the limit

$$
C_{g} \sim|d| \sim|\mu| \sim L^{-1} \ll 1, \quad k \sim \log L
$$

and treat logarithmically large terms (in particular, $k$ ) as $\mathrm{O}(1)$. In fact, when taking into account the numerical values of the parameters that are either of order unity or logarithmically large, assumptions (35) become

$$
\delta \sim|d| \sim\left|\alpha_{5} \mu\right| \sim \frac{v_{\mathrm{g}}}{L} \ll 1 .
$$

As is usually the case, the large depth limit only requires in practice that $\exp (-2 k)$ be numerically small, which is already satisfied at $k=2$, even though the wavelength of the surface waves is $2 \pi / k=\pi>1=\mathrm{depth}$ in this case. 


\section{Coupled amplitude-mean flow (CAMF) equations}

In the limit (35) the container depth is logarithmically large compared with the basic wavelength. The parameters $\alpha_{7}, \beta_{3}$ and $\beta_{4}$ are small compared to one (sec (31) and (33)) and can be set to () in the GCAMF equations. In particular, the right-hand side of the third boundary condition of (22) disappears and, since this was responsible for the strong dependence of the mean flow in $x$, we may assume that the mean flow depends slowly on $x$, i.c.

$$
\left|f_{x x}^{n}\right| \ll\left|f_{x}^{m}\right| \ll\left|f^{m}\right| \ll 1, \quad\left|\psi_{x x}^{m}\right| \ll\left|\psi_{x}^{m}\right| \ll\left|\psi^{m}\right| \ll 1 . \quad\left|\Omega_{x x}^{m}\right| \ll\left|\Omega_{x}^{m}\right| \ll\left|\Omega^{m}\right| \ll 1 .
$$

If in addition we anticipate that

$$
\left|A^{ \pm}\right|^{2} \sim\left|f^{m}\right| \sim\left|\psi_{r}^{m}\right| \sim\left|\Omega^{m}\right| \sim L^{-1} \ll 1,
$$

then convective terms are small compared to diffusive terms in the second momentum condition of Eq. (20) and can be neglected. Using all these, the GCAMF equations (18)-(24) can be simplified to

$$
\begin{aligned}
& A_{t}^{ \pm} \mp v_{\mathrm{g}} A_{x}^{ \pm}=\mathrm{i} \alpha A_{x x}^{ \pm}-(\delta+\mathrm{i} d) A^{ \pm}+\mathrm{i}\left(\alpha_{3}\left|A^{ \pm}\right|^{2}-\alpha_{4}\left|A^{\mp}\right|^{2}\right) A^{ \pm}+\alpha_{5} \mu \bar{A}^{\mp} \pm \mathrm{i} \alpha_{6} \int_{-1}^{0} g(y) \psi_{y}^{n t} \mathrm{~d} y A^{ \pm}, \\
& A^{ \pm}(x+L, t) \equiv \Lambda^{ \pm}(x, t)
\end{aligned}
$$

and

$$
\Omega^{n}=\psi_{y y}^{m}, \quad \Omega_{t}^{m}=C_{g} \Omega_{y y}^{m},
$$

in $-1<y<0$, with boundary conditions

$$
\begin{aligned}
& \psi_{x}^{m}-\int_{i}^{m}=\beta_{1}\left(\left|A^{-}\right|^{2}-\left|A^{+}\right|^{2}\right)_{x}, \quad \psi_{y y}^{m}=\beta_{2}\left(\left|A^{+}\right|^{2}-\left|A^{-}\right|^{2}\right), \\
& (1-S) \int_{x}^{m}-S f_{x x x}^{m}-\psi_{y t}^{m}+C_{g} \psi_{y y y}^{m}=0 \quad \text { at } y=0, \\
& \int_{0}^{L} \Omega_{y}^{m} \mathrm{~d} x=\psi^{m}=\psi_{y}^{m}=0 \quad \text { at } y=-1, \\
& \psi^{m}(x+L, y, t) \equiv \psi^{m}(x, y, t), \quad f^{m}(x+L, t) \equiv f^{m}(x, t), \\
& \int_{0}^{L} f^{m}(x, t) \mathrm{d} x=0,
\end{aligned}
$$

where we have taken into account that, according to (37)

$$
\left\langle\psi_{y}^{m}\right\rangle^{x} \equiv \psi_{y}^{m}
$$

in first approximation. In addition, the inviscid dispersion relation (8) can be approximated as

$$
\omega(k)=\left[\left(1-S+S k^{2}\right) k\right]^{1 / 2}
$$

and Eqs. (26)-(33) simplify to

$$
\begin{aligned}
& \delta=2 k^{2} C_{g}, \quad d=-\left(\frac{2 \pi N}{L}-k_{0}\right) v_{\mathrm{g}}, \quad v_{\mathrm{g}}=\omega^{\prime}(k), \quad \alpha=-\frac{\omega^{\prime \prime}(k)}{2}, \\
& \alpha_{3}=\frac{3 S \omega k^{4}}{1-S-2 S k^{2}}+\frac{\left[8(1-S)+5 S k^{2}\right] \omega k^{2}}{4\left(1-S+S k^{2}\right)}, \quad \alpha_{4}=\frac{\omega k^{2}}{2}\left[\frac{4\left(1-S+S k^{2}\right)}{1-S+4 S k^{2}}+\frac{4(1-S)+7 S k^{2}}{1-S+S k^{2}}\right],
\end{aligned}
$$




$$
\begin{aligned}
& \alpha_{5}=\omega k, \quad \alpha_{6}=\frac{k}{2 \omega}, \quad \beta_{1}=2 \omega, \quad \beta_{2}=8 \omega k^{2}, \\
& g(y)=4 \omega k \exp (2 k y) .
\end{aligned}
$$

For convenience we use the re-scaled variables

$$
A=\frac{A^{+}}{C_{g}^{1 / 2}}, \quad B=\frac{A^{-}}{C_{g}^{1 / 2}}, \quad \varphi=\frac{\psi^{m}}{C_{g}}, \quad F=f^{m} L, \quad \tau=C_{g} l, \quad \xi=\frac{x}{L},
$$

which are of order 1 in the limit we are considering here, to rewrite (39)-(44) as

$$
\begin{aligned}
& \Lambda_{\tau}-\Lambda \Lambda_{\xi}=\mathrm{i} \varepsilon \alpha \Lambda_{\xi \xi}-\left(2 k^{2}+\mathrm{i} \tilde{d}\right) A+\mathrm{i}\left(\alpha_{3}|A|^{2}-\alpha_{4}|B|^{2}\right) \Lambda+\tilde{\mu} \bar{B}+2 \mathrm{i} k^{2} \int_{-1}^{0} \mathrm{e}^{2 k y} \varphi_{y} \mathrm{~d} y \Lambda, \\
& B_{t}+\Lambda B_{\xi}=\mathrm{i} \varepsilon \alpha B_{\xi \xi}-\left(2 k^{2}+\mathrm{i} \tilde{d}\right) B+\mathrm{i}\left(\alpha_{3}|B|^{2}-\alpha_{4}|A|^{2}\right) B+\tilde{\mu} \bar{A}-2 \mathrm{i} k^{2} \int_{-1}^{0} \mathrm{e}^{2 k y} \varphi_{y} \mathrm{~d} y B \\
& \varphi_{y y \tau}=\varphi_{y y y}
\end{aligned}
$$

in $-1<y<0$, with boundary conditions

$$
\begin{aligned}
& \varphi_{\xi}-F_{\tau}=2 \omega\left(|B|^{2}-|A|^{2}\right)_{\xi}, \quad \varphi_{y y}=8 \omega k^{2}\left(|A|^{2}-|B|^{2}\right), \quad \varphi_{y y y}-\varphi_{y \tau}+I F_{\xi}=0 \quad \text { at } y=0, \\
& \int_{0}^{1} \varphi_{y y} \mathrm{~d} \xi=\varphi=\varphi_{y}=0 \quad \text { at } y=-1, \\
& A(\xi+1, \tau) \equiv A(\xi, \tau), \quad B(\xi+1, \tau) \equiv B(\xi, \tau), \\
& \varphi(\xi+1, y, \tau) \equiv \varphi(\xi, y, \tau), \quad F(\xi+1, \tau) \equiv F(\xi, \tau), \\
& \int_{0}^{1} F(\xi, \tau) \mathrm{d} \xi=0,
\end{aligned}
$$

where the re-scaled forcing amplitude and detuning are

$$
\tilde{\mu}=\frac{\mu \omega k}{C_{g}}, \quad \tilde{d}=\frac{d}{C_{g}} .
$$

These are the control parameters of the system, and can be selected by means of the forcing amplitude and (slight variations of) the forcing frequency. Invoking (11) and (46), the control parameters are subject to the following limitations

$$
\tilde{\mu}>0, \quad-\Lambda \pi<\tilde{d} \leq \Lambda \pi .
$$

In addition, the problem depends on $\omega, k, \alpha_{3}, \alpha_{4}$ and the following additional parameters

$$
\Lambda=\frac{v_{\mathrm{g}}}{C_{g} L} \sim 1, \quad \Gamma=\frac{1-S}{\left(C_{g} L\right)^{2}} \sim 1, \quad \varepsilon=\frac{1}{C_{g} L^{2}} \ll 1 .
$$

These latter parameters are readily calculated, using also (45)-(47), in terms of $\omega, S, C_{g}$ and $L$.

The remaining part of the paper is devoted to the analysis of Eqs. (51)-(57). But some remarks about these are now in order.

- We are including higher order terms in (51) and (52) that result from dispersion. As we shall see in Section 4 , dispersive terms cannot be ignored a priori because they can produce intermediate scales that are not present in the hyperbolic approximation of (51) and (52), obtained when $\varepsilon$ is set to 0 [20]. 
- The streamfunction of the mean flow $\varphi$ can be seen as resulting from a Eulerian temporal mean of the original streamfunction $\psi$ in the shortest timescalc. For convenience we also consider the streamfunction obtained by a Lagrangian mean. The associated velocity $\left(u^{\mathrm{mt}}, v^{\mathrm{mt}}\right)$ is also called mass iransport velocity [21], and equals the Eulcrian velocity plus the Stokes drift [22]. Bolh are purcly hori $/ 0 n t a l$ in first approximation, and

$$
\left.u^{\mathrm{mt}}=-\left[\varphi_{y}^{m}+(\mid \Lambda]^{2}-|B|^{2}\right) g(y)\right] .
$$

Note that the Eulerian mean flow is of the same order as the Stokes drift, although the former is usually ignored in the analysis of convection of passive scalars in the Faraday system at low viscosity $[23,24]$, which should not be done.

- The mean flow is forced by the surface waves in two ways. They produce a normal velocity at the free surface (right-hand side of the first condition of (54)), which is responsible for the iniscid mean flow in the DaveyStewartson equations [25], and also a shear stress forcing (right-hand side of the second condition of (54)) that involves viscous effects in an essential way.

- Eqs. (51)-(57) are invariant under the following actions

$$
\begin{aligned}
& \varphi \rightarrow-\varphi, \quad A \leftrightarrow B, \quad \xi \rightarrow-\xi ; \\
& A \rightarrow \mathrm{c}^{-\mathrm{i} c_{1}} A, \quad B \rightarrow \mathrm{c}^{\mathrm{i} c_{1}} B ; \quad \text { and } \quad \xi \rightarrow \xi+c_{2},
\end{aligned}
$$

which come from invariance of the original equations (1)-(4) under horizontal translation and reflection. Note that irvariance under translation splits into two actions, which are not expected to apply independently at higher order. Thus some additional higher order terms should be added to break this spurious symmetry. These could affect the dynamics in a still slower timescale, which is ignored here.

- The simplest reflection symmetric solutions of (51)-(57) are the spatially uniform SWs without any mean flow, $|A|=|B|=$ constant, $\varphi=F=0$, analyzed in Section 4 . It can be seen that these are the only possible attractors of (51)-(57) without mean flow; any other wave pattern will involve a nonzero mean flow.

\section{Spatially uniform SWs and their linear stability}

Eqs. (51)-(57) admit the flat steady state $A=B=\varphi=F=0$, which is stable only if

$$
\tilde{\mu} \leq \tilde{\mu}_{0} \equiv \sqrt{4 k^{4}+\tilde{d}^{2}}
$$

This marginal instability curve is the typical resonance tonguc associated with parametrically excited waves [5]. At $\tilde{\mu}=\tilde{\mu}_{0}$ there is a pitchfork bifurcation to a new branch of spatially uniform steady states, which correspond to spatially uniform SWs of the original problem. In fact, there are infinitely many branches of these, which are of the form

$$
\Lambda=\Lambda_{m} \equiv R_{m} \mathrm{e}^{\mathrm{i} 0_{m}+\mathrm{i} 2 \pi m \xi}, \quad B=B_{m} \equiv R_{m} \mathrm{e}^{\mathrm{i} \phi_{m}-\mathrm{i} 2 \pi m \xi}, \quad \varphi=F=0
$$

with $R_{m} \geq 0, \theta_{m}$, and $\phi_{m}$ given by

$$
\begin{aligned}
& {\left[R_{m}^{2}\left(\alpha_{4}-\alpha_{3}\right)+\tilde{d}_{m}\right]^{2}+4 k^{4}=\tilde{\mu}^{2}, \quad \cos \left(\theta_{m}+\phi_{m}\right)=\frac{2 k^{2}}{\tilde{\mu}},} \\
& \sin \left(\theta_{m}+\phi_{m}\right)=-\frac{R_{m}^{2}\left(\alpha_{4}-\alpha_{3}\right)+\tilde{d}_{m}}{\tilde{\mu}}
\end{aligned}
$$

in terms of the extended detuning

$$
\tilde{d}_{m}=\tilde{d}-2 \pi m \Lambda
$$




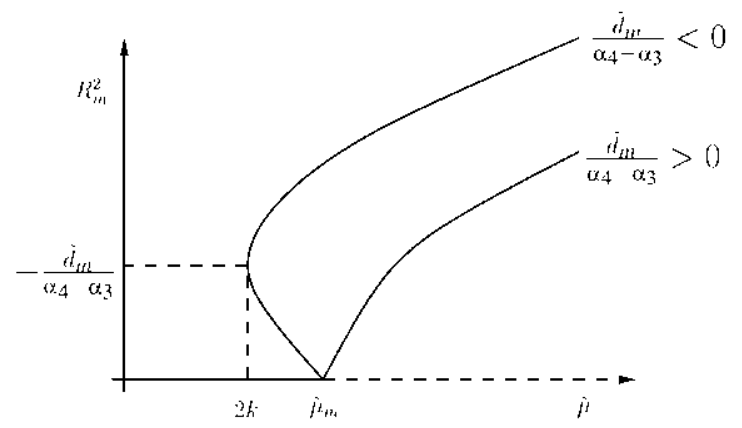

Fig. 2. Bifurcation diagram of the spatially uniform SWs, given by (65).

and bifurcate from the flat state at

$$
\tilde{\mu}=\tilde{\mu}_{m} \equiv \sqrt{4 k^{4}+(\tilde{d}-2 \pi m \Lambda)^{2}} \text { for each integer } m .
$$

According to (59) and (65), $\tilde{\mu}_{m}>\tilde{\mu}_{0}$ if $m \neq 0$ and the bifurcation at $\tilde{\mu}=\tilde{\mu}_{m}$ is supercritical if $\tilde{d}_{m} /\left(\alpha_{4}-\alpha_{3}\right) \geq 0$ and subcritical otherwise. In the latter case, the bifurcated branch is C-shaped (Fig. 2), with a saddle-node at

$$
R_{m}^{2}=-\frac{\tilde{d}_{m}}{\alpha_{4}-\alpha_{3}} .
$$

The linear stability of these steady states is analyzed by first rewriting Eqs. (51)-(57) in terms of the new variables $a$ and $b$, defined as

$$
A=A_{m}(1+a), \quad B=B_{m}(1+b)
$$

and then linearizing around $a=b=\varphi=F=0$. For convenience we use the new variables

$$
r^{ \pm}=(a+\bar{a}) \pm(b+\bar{b}), \quad s^{ \pm}=(a-\bar{a}) \pm(b-\bar{b})
$$

to rewrite the resulting linear problem, after some algebra, as

$$
\begin{aligned}
& r_{\tau}^{+}-\Lambda r_{\xi}^{-}=\mathrm{i} \varepsilon \alpha s_{\xi \xi}^{+}+2 \mathrm{i}\left[R_{m}^{2}\left(\alpha_{3}-\alpha_{4}\right)-\tilde{d}_{m}\right] s^{+}, \\
& r_{\tau}^{-}-\Lambda r_{\xi}^{+}=\mathrm{i} \varepsilon \alpha s_{\xi \xi}^{-}-4 k^{2} r^{-}, \\
& s_{\tau}^{+}-\Lambda s_{\xi}^{-}=\mathrm{i} \varepsilon \alpha r_{\xi \xi}^{+}-4 k^{2} s^{+}+2 \mathrm{i} R_{m}^{2}\left(\alpha_{3}-\alpha_{4}\right) r^{+}, \\
& s_{\tau}^{-}-\Lambda s_{\xi}^{+}=\mathrm{i} \varepsilon \alpha r_{\xi \xi}^{-}+2 \mathrm{i}\left(R_{m}^{2} \alpha_{3}-\tilde{d}_{m}\right) r^{-}+8 \mathrm{i} k^{2} \int_{-1}^{0} \mathrm{e}^{2 k y} \varphi_{y} \mathrm{~d} y, \\
& \varphi_{y y \tau}=\varphi_{y w y} \quad \text { in }-1<y<0, \\
& \varphi_{\xi}-F_{\tau}-2 \omega R_{m}^{2} r_{\xi}^{-}=\varphi_{y y}-8 \omega k^{2} R_{m}^{2} r_{\xi}^{-}=\varphi_{m y}-\varphi_{y \tau}+\Gamma F_{\xi}=0 \quad \text { at } y=0, \\
& \int_{0}^{1} \varphi_{m y} \mathrm{~d} \xi=\varphi=\varphi_{y}=0 \quad \text { at } y=-1, \\
& r^{ \pm}(\xi+1, \tau)=r^{ \pm}(\xi, \tau), \quad s^{ \pm}(\xi+1, \tau)=s^{ \pm}(\xi, \tau), \\
& \varphi(\xi+1, y, \tau)=\varphi(\xi, y, \tau), \quad F(\xi+1, \tau)=F(\xi, \tau),
\end{aligned}
$$




$$
\int_{0}^{1} F(\xi, \tau) \mathrm{d} \xi=0 .
$$

In order to proceed we must distinguish two different cases depending on the relative size of the wavelength of the perturbations compared to the small dispersive length $\sqrt{\varepsilon}$, see $(69)-(72)$.

\subsection{Long-wave perturbations}

These perturbations exhibit a wavelength that is large compared to $\varepsilon^{1 / 2}$. Thus dispersive terms are small in (69)-(72) and can be neglected. We seek normal modes of (69)-(77), of the form

$$
\left(r^{ \pm}, s^{ \pm}, \varphi, F\right)=\left(r_{n}^{ \pm}, s_{n}^{ \pm}, \varphi_{n}(y), F_{n}\right) \mathrm{e}^{\lambda \tau+\mathrm{i} 2 \pi n \xi} \quad \text { with } n=\text { integer } \ll \varepsilon^{-1 / 2}
$$

and obtain the dispersion relation as follows. From (73)-(77) we calculate

$$
\varphi_{n}=\omega\left(D_{1 n}[\cosh (\sqrt{\lambda}(y+1))-1]+D_{2 n}[\sinh (\sqrt{\lambda}(y+1))-\sqrt{\lambda}(y+1)]\right) R_{m}^{2} r_{n}^{-},
$$

where

$$
\begin{aligned}
& D_{1 n}=\frac{8 k^{2} \lambda^{2} \sqrt{\lambda}+8 \pi^{2} n^{2} \Gamma\left[4 k^{2} \sqrt{\lambda}-\left(\lambda+4 k^{2}\right) \sinh \sqrt{\lambda}\right]}{\lambda^{2} \sqrt{\lambda} \cosh \sqrt{\lambda}+4 \pi^{2} n^{2} \Gamma(\sqrt{\lambda} \cosh \sqrt{\lambda}-\sinh \sqrt{\lambda})}, \\
& D_{2 n}=\frac{8 \pi^{2} n^{2} \Gamma\left[\left(\lambda+4 k^{2}\right) \cosh \sqrt{\lambda}-4 k^{2}\right]}{\lambda^{2} \sqrt{\lambda} \cosh \sqrt{\lambda}+4 \pi^{2} n^{2} \Gamma(\sqrt{\lambda} \cosh \sqrt{\lambda}-\sinh \sqrt{\lambda})},
\end{aligned}
$$

and invoking (78) we obtain the integral appearing in that term accounting for coupling with the streaming flow in (72) as $\int_{-1}^{0} \mathrm{e}^{2 k y} \varphi_{n y} \mathrm{~d} y=\omega R_{m}^{2} r_{n}^{-} D_{n}(\lambda)$, where

$$
\begin{aligned}
D_{n}= & \frac{2 k \sinh \sqrt{\lambda}-\sqrt{\lambda} \cosh \sqrt{\lambda}+\sqrt{\lambda} \mathrm{e}^{-2 k}}{\sqrt{\lambda}\left(4 k^{2}-\lambda\right)} D_{1 n} \\
& +\frac{2 k \cosh \sqrt{\lambda}-\sqrt{\lambda} \sinh \sqrt{\lambda}-2 k+\lambda\left(1-\mathrm{e}^{-2 k}\right) /(2 k)}{\sqrt{\lambda}\left(4 k^{2}-\lambda\right)} D_{2 n} .
\end{aligned}
$$

Note that $\mathrm{e}^{-2 k}$ should be neglected according to (35), but we do not do that to avoid a spurious singularity at $\sqrt{\lambda}=2 k$. And we only necd to use Eqs. (69)-(72) to obtain

$$
\begin{aligned}
& \lambda\left(\lambda+4 k^{2}\right)\left[\lambda\left(\lambda+4 k^{2}\right)+2(2 \pi n \Lambda)^{2}\right]-4 \lambda\left(\lambda+4 k^{2}\right) R_{m}^{2}\left(\alpha_{3}-\alpha_{4}\right)\left[\tilde{d}_{m}+R_{m}^{2}\left(\alpha_{4}-\alpha_{3}\right)\right]+(2 \pi n \Lambda)^{4} \\
& -4(2 \pi n \Lambda)^{2}\left[\tilde{d}_{m}+R_{m}^{2}\left(\alpha_{4}-\alpha_{3}\right)\right]\left(\tilde{d}_{m}-2 R_{m}^{2}\left[\alpha_{3}+2 \omega k^{2} D_{n}(\lambda)\right]\right)=0 .
\end{aligned}
$$

Now we analyze this dispersion relation, with some emphasis on the effect of the mean flow, which comes through $D_{n}(\lambda)$.

Under spatially uniform perturbations, $n=0$, marginal instability occurs as

$$
R_{m b}^{2}=\frac{\tilde{d}_{m}}{\alpha_{3}-\alpha_{4}},
$$

which corresponds to the saddle-node in Fig. 2. This instability yields the straight line labeled $\Lambda_{1}$ in Fig. 3.

Under spatially nonuniform perturbations $(n \neq 0)$ marginal instability appears as either:

(a) $\lambda=0$ (steady instability), which occurs as

$$
\left[\tilde{d}_{m}+R_{m}^{2}\left(\alpha_{4}-\alpha_{3}\right)\right]\left[\tilde{d}_{n}-2 R_{m}^{2}\left(\alpha_{3}+2 \omega k^{2} \mathcal{D}_{1}\right)\right]-\frac{(2 \pi n \Lambda)^{2}}{4}>0
$$



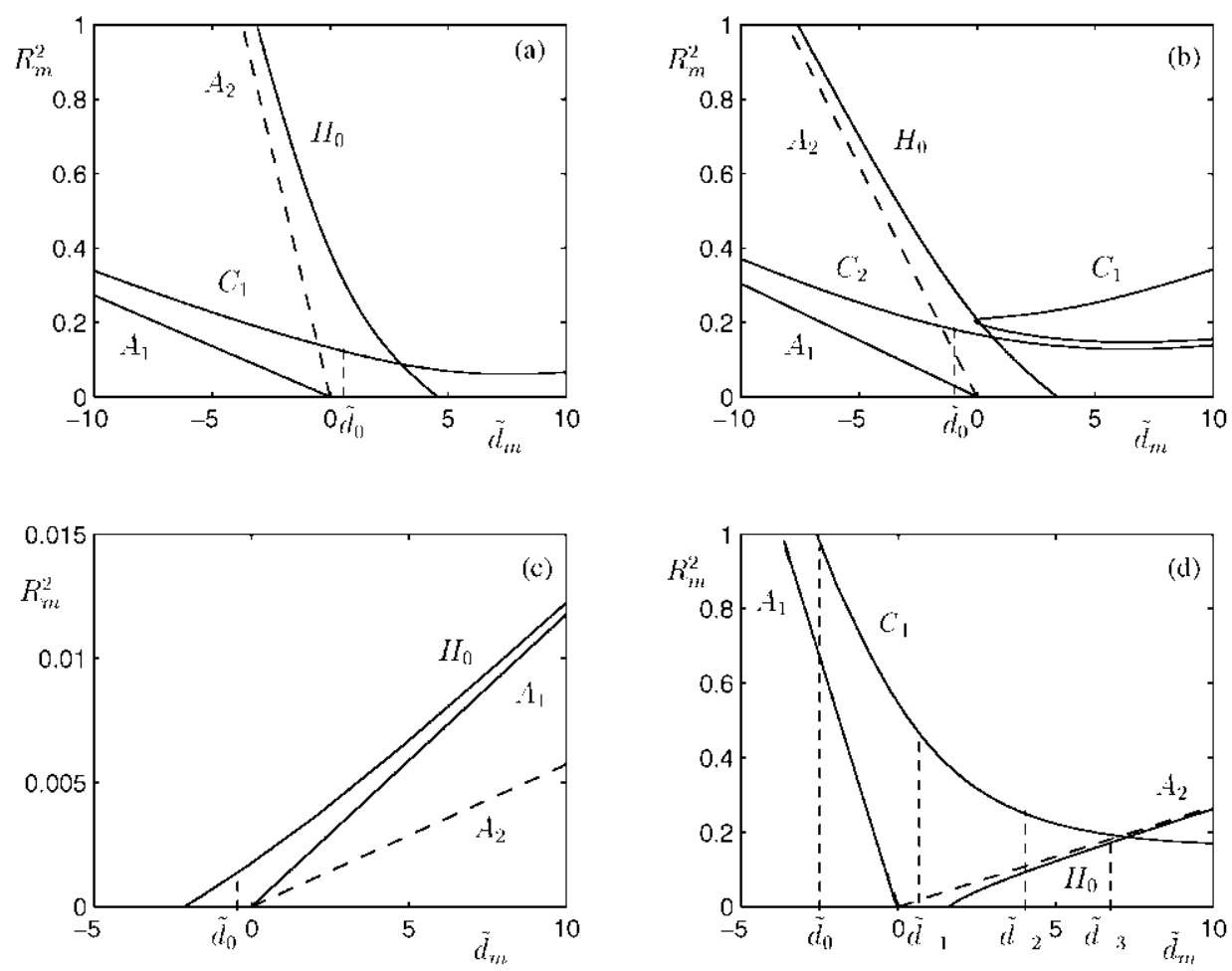

Fig. 3. Stability diagram for the spatially uniform $S W s$ under long-wave perturbations for $\Gamma=1, k=2$ and (a) $S=0.5$. (b) $S=0.29$, (c) $S=0.11$, (d) $S=0.05$. The stable region is that in between of the axis $R_{m}=0$, the straight line $A_{1}$, and the curves $H_{0}$ and either $C_{1}$ (in cases a. $c$ and d) or $C_{2}$ (in case b).

where

$$
\mathcal{D}_{1} \equiv \mathcal{D}_{n}(0)=\frac{\left(3+2 k^{2}\right)\left(1-2 k-\mathrm{e}^{-2 k}\right)}{2 k^{2}}+\frac{3\left(1+2 k^{2}\right)\left(1-2 k+2 k^{2}-\mathrm{e}^{-2 k}\right)}{4 k^{3}} .
$$

Note that we have infinitely many marginal instability curves, one for each integer $n$. But the most dangerous instability is seen to be that for $n=1$, which is the hyperbola labeled $H_{0}$ in Fig. 3, whose asymptotes are $A_{1}$ (given by (84)) and $A_{2}$, which is given by

$$
R_{m}^{2}=\frac{\tilde{d}_{m}}{2\left(\alpha_{3}+2 \omega k^{2} \mathcal{D}_{1}\right)} .
$$

Depending on the relative position of $A_{1}$ and $A_{2}$, we can have four qualitatively different configurations of the marginal instability curves $A_{1}$ and $H_{0}$, which are like those plotted in Fig. 3a, c and d (Fig. 3a and b are qualitatively similar), and the one obtained from Fig. 3 a under the reflection symmetry $\tilde{d}_{m} \rightarrow-\tilde{d}_{m}$; here we take into account that the various coefficients appearing in (83) are not independent, see Eqs. (46)-(49) and (60). This well documented instability [4] yields stationary, spatially nonuniform complex amplitudes like those in Fig. 10a.

(b) $\lambda=\mathrm{i} \Omega \neq 0$ (oscillatory instability), which occurs as

$$
R_{m}^{4}=\frac{E_{n}(\Omega)^{2}}{G_{n}(\Omega)}, \quad \tilde{d}=\frac{E_{n}(\Omega)}{R_{m}^{2}}-R_{m}^{2}\left(\alpha_{4}-\alpha_{3}\right)
$$


for certain functions $E_{n}$ and $G_{n}$ that are not given here for the sake of brevity. These two equations come from the real and the imaginary parts of the dispersion relation, and provide the curves labeled $C_{n}$ in Fig. 3, which always have the asymptote $A_{1}$; other asymptotes are associated with singularitics of the function $E_{n}$. We do not try to classify the several relative positions of these curves, as we did in case (a) above, because now the classification is too involved. This oscillatory instability involves a double eigenvalue (associated with $\pm n$, because (83) depends on the wavenumber $n$ through its square, $n^{2}$ ), and yiclds cither symmetric or nonsymmetric patterns, which could be seen as either traveling waves (TWs) or SWs for the wave envelopes, like those in Figs. 6a and 8a below, sec $[4,26]$ and references therein. Note nevertheless that the resulting reconstructed patterns for, e.g., the free surface elevation using Eq. (96) below are much more involved than TWs or SWs.

Some remarks are now in order:

(i) The three instabilities considered above (namely, saddle-node, steady, and oscillatory, see Eqs. (84), (85) and (87)) are usually present.

(ii) If the contribution of the mean flow to amplitude equations is not considered (namely, if $D_{n}(\lambda)$ is set to 0 in (83)), then the curves $C_{n}$ that yicld oscillatory instabilities disappear. Thus the mean flow drastically reduces the stability region.

(iii) The oscillatory instability is usually the one giving the first instability of the spatially uniform SWs as the forcing amplitude is increased.

(iv) The most unstable mode at threshold is frequently (but not always, see Fig. 3b) the first mode, $n=1$.

\subsection{Short-wave perturbations}

These are associated with dispersion, exhibit a wavenumber of order $\varepsilon^{-1 / 2}$, and in first approximation, propagate with the group velocity $\Lambda$, see (69)-(72). Thus we seek normal modes of (69)-(77) of the form

$$
\begin{aligned}
& \left(r^{ \pm}, s^{ \pm}\right)=\left[\left(r_{0}^{ \pm}, s_{0}^{ \pm}\right)+\sqrt{\varepsilon}\left(r_{1}^{ \pm}, s_{1}^{ \pm}\right)+\cdots\right] \mathrm{e}^{\left(\lambda_{0} / \sqrt{\varepsilon}+\lambda_{1}+\cdots\right) \tau+\mathrm{i} K \xi / \sqrt{\varepsilon},} \\
& (\varphi, F)=\left[\left(\varphi_{0}(y), F_{0}\right)+\sqrt{\varepsilon}\left(\varphi_{1}(y), F_{1}\right)+\cdots\right] \mathrm{e}^{\left(\lambda_{0} / \sqrt{ } \varepsilon+\lambda_{1}+\cdots\right) \tau+\mathrm{i} K \xi / \sqrt{ } \varepsilon},
\end{aligned}
$$

where $K \sim 1$. Replacing these into (69)-(72) we obtain, at orders $\mathrm{O}(\varepsilon)^{-1 / 2}$ and $\mathrm{O}(1)$

$$
\begin{aligned}
& \lambda_{0}= \pm \mathrm{i} \Lambda K, \quad r_{0}^{-}= \pm r_{0}^{+}, \quad s_{0}^{-}= \pm s_{0}^{+}, \\
& \varphi_{0}=\frac{2 I^{\top} \omega R_{m}^{2} r_{0}^{-}(y+1)}{\Gamma-\Lambda^{2}}, \quad F_{0}=\frac{ \pm \Lambda \varphi_{0}(0)}{\Gamma-\Lambda^{2}},
\end{aligned}
$$

and

$$
\begin{aligned}
& \mathrm{i} \Lambda K\left( \pm r_{1}^{+}-r_{1}^{-}\right)=-\lambda_{1} r_{0}^{+}-\mathrm{i}\left[K^{2} \alpha-2 R_{m}^{2}\left(\alpha_{3}-\alpha_{4}\right)+2 \tilde{d}_{m}\right] s_{0}^{+}, \\
& \mathrm{i} \Lambda K\left( \pm r_{1}^{-}-r_{1}^{+}\right)=-\left(\lambda_{1}+4 k^{2}\right) r_{0}^{-}-\mathrm{i} K^{2} \alpha s_{0}^{-}, \\
& \mathrm{i} \Lambda K\left( \pm s_{1}^{+}-s_{1}^{-}\right)=-\left(\lambda_{1}+4 k^{2}\right) s_{0}^{+}-\mathrm{i}\left[\alpha K^{2}-2 R_{m}^{2}\left(\alpha_{3}-\alpha_{4}\right)\right] r_{0}^{+}, \\
& \mathrm{i} \Lambda K\left( \pm s_{1}^{-}-s_{1}^{+}\right)=-\lambda_{1} s_{0}^{-}-\mathrm{i}\left[\alpha K^{2}-4 R_{m}^{2} \alpha_{3}+2 \tilde{d}_{m}-8 R_{m}^{2} \omega D_{2}\right] r_{0}^{-},
\end{aligned}
$$

where

$$
\mathcal{D}_{2}=-\Gamma\left[k\left(\Gamma-\Lambda^{2}\right)\right]^{-1}
$$

is the limit, as $\lambda \simeq 2 \mathrm{i} \pi n \Lambda$ and $n \rightarrow \infty$, of $D_{n}(\lambda)$ (after neglecting $\mathrm{O}\left(\mathrm{e}^{-2 k}\right)$-1crms). Note that $\mathcal{D}_{2}$ diverges at the (cxcluded) resonance $I=A^{2}$, which occurs when the group velocity $A$ coincides with the (re-scaled) phase 
velocity of the inviscid long wave modes $\Gamma^{1 / 2}$. And we only need to require that Eqs. (90)-(93) possess nontrivial solutions, invoking (88), to obtain the asymptotic dispersion relation

$$
\left(\lambda_{1}+2 k^{2}\right)^{2}+\left[\tilde{d}_{m}+\alpha K^{2}-R_{m}^{2}\left(2 \alpha_{3}-\alpha_{4}+2 \omega k^{2} \mathcal{D}_{2}\right)\right]^{2}=R_{m}^{4}\left(\alpha_{3}+2 \omega k^{2} \mathcal{D}_{2}\right)^{2} .
$$

Marginal instability is readily seen to only occur along the curve $\lambda_{1}=0$, which yields

$$
R_{m}^{4}\left(\alpha_{3}+2 \omega k^{2} \mathcal{D}_{2}\right)^{2}=\left[\tilde{d}_{m}+\alpha K^{2}-R_{m}^{2}\left(2 \alpha_{3}-\alpha_{4}+2 \omega k^{2} \mathcal{D}_{2}\right)\right]^{2}+4 k^{4} .
$$

The instability region in the plane $R_{m}^{2}$ vs. $\tilde{d}_{m}$ is the envelope of the curves (95) as $K$ varies from 0 to $\infty$. This is obtained upon translation of the curve for $K=0, H^{\infty}$, towards positive $\tilde{d}_{m}$ if $\alpha<0$ and towards negative $\tilde{d}_{m}$ if $\alpha>0$. Note that $H^{\infty}$ exhibits two asymptotes, $A_{1}$ (given by (84)) and $A_{3}$, which is given by

$$
R_{m}^{2}=\frac{\tilde{d}_{m}}{\alpha_{3}-\alpha_{4}+2\left(\alpha_{3}+2 \omega k^{2} \mathcal{D}_{2}\right)} .
$$

The curve $H^{\infty}$ is qualitatively similar to either one of those plotted in Fig. 4 or those obtained from these by a reflection symmetry $\tilde{d}_{m} \rightarrow-\tilde{d}_{m}$; in particular, that for $S=0.5$ is qualitatively similar to that in Fig. 4 a, but with the asymptotes $A_{1}$ and $A_{3}$ so close to each other that the curve is hardly seen in any reasonable plot.

This is again an oscillatory instability with a nonzero wavenumber. The involved short-wave wavetrains counterpropagate with the group velocity $\Lambda$ and, as in Section 4.1 (case b), the bifurcated solutions are either symmetric or nonsymmetric for the wave envelopes, like those in Figs. $5 \mathrm{a}$ and $7 \mathrm{a}$ below.
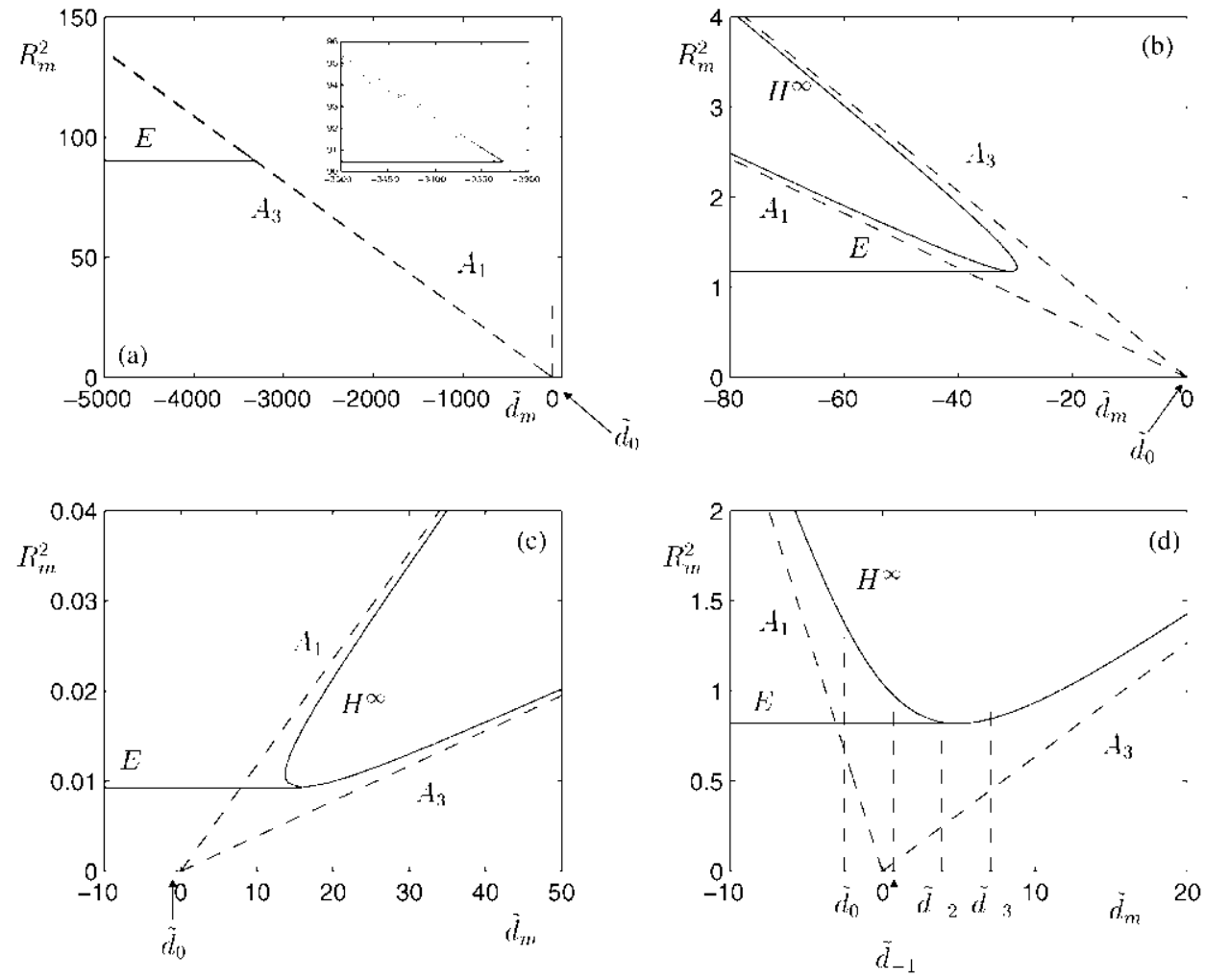

Fig. 4. Stability diagram for the spatially uniform $S W s$ under short-wave perturbations for $\Gamma=1, k=2$ and (a) $S=0.5$. (b) $S=0.29$. (c) $S=0.11$ and (d) $S=0.05$. The stable region is that below the minimum of the curve $H^{x}$ and the straight line $E$. 
$|.4|$

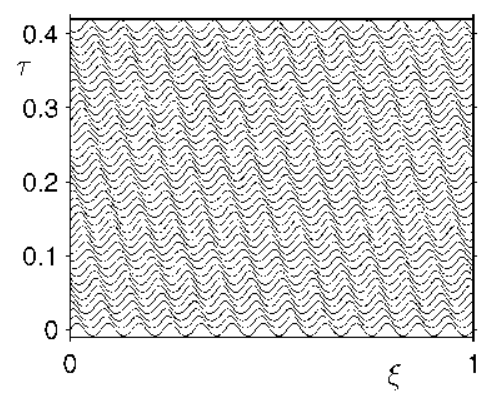

$|A|$

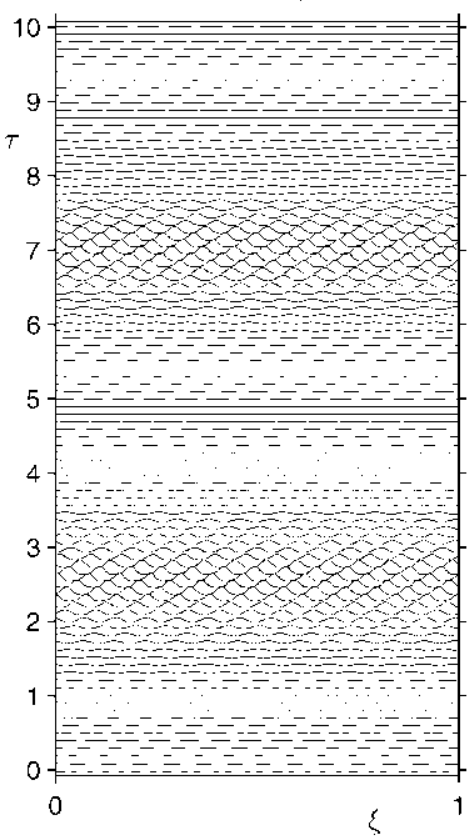

(a)

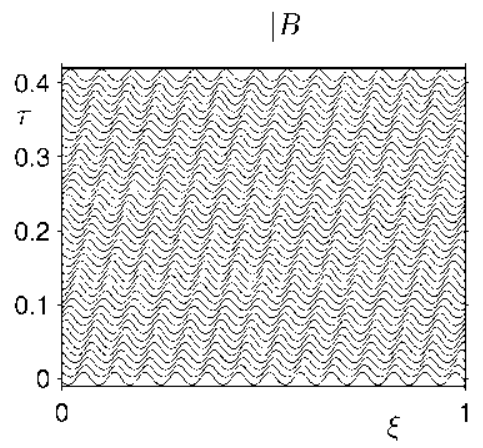

$\mid B$

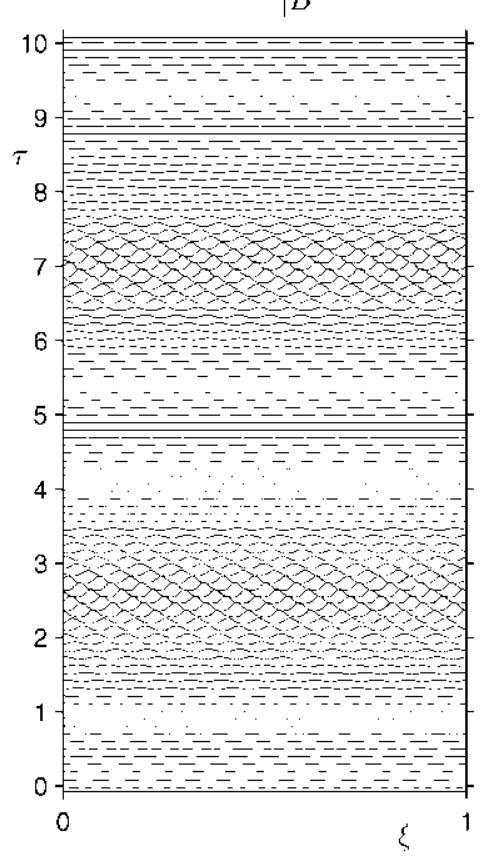

Fig. 5. Space-time plots of $|A|$ and $|B|$ for some attractors at $C_{g} L=1, \varepsilon=5 \times 10^{3}, S=0.05, \tilde{d}=-2.5$, and (a) $\tilde{\mu}=8.04$ and (b) $\tilde{\mu}=8.12$.

\section{Large-time dynamics beyond threshold}

In this section we numerically integrate (51)-(57) to explore the attractors that appear when the forcing amplitude is increased above the SW stability limits calculated in the previous section. As representative cases, we consider those indicated with vertical dashed lines in Figs. 3 and 4, in which the first instability of the spatially uniform SWs (64), with wavenumber $m=0$ is (A) a short-wave instability, (B) a long wave, oscillatory instability with a wavenumber $n= \pm 1$, (C) a long wave, oscillatory instability with a wavenumber $n= \pm 2$, and (D) a long wave, steady instability (which always occurs with $n= \pm 1$ ). For simplicity, all patterns are described below in terms of the complex amplitudes $A$ and $B$. The reconstructed pattern for, e.g., the free surface elevation is obtained using (13) and (50), to be

$$
f=C_{g}^{1 / 2}\left[A \mathrm{e}^{\mathrm{i}(\omega t+k x)}+B \mathrm{e}^{\mathrm{i}(\omega t-k x)}+\text { c.c. }\right]+\cdots
$$


Case A At $S=0.05$ and $\tilde{d}=-2.5$, we have $\tilde{d}_{0}=-2.5$ (see (66)), and the primary instability of the spatially uniform SW given by (64) with $m=0$ is dispersive, see Figs. $3 \mathrm{~d}$ and $4 \mathrm{~d}$. Thus the primary bifurcated patterns for the wave envelopes at threshold, $\tilde{\mu}=8$, are reflection symmetric pairs of dispersive wavetrains (one of the two possibilities anticipated at the end of Section 4.2), like that in Fig. 5a. Note that the two counterpropagating wavetrains travel at the group velocity, which is $\Lambda \simeq 0.51$ in the present case. In the range $8.03<\tilde{\mu}<8.13$, various oscillatory instabilities take place that yield quasi-periodic waves; one of them exhibiting a near-homoclinic behavior is plotted in Fig. 5b. At $\tilde{\mu}=8.13$ the system jumps to a spatially uniform SW with wavenumber $m=-1$, which is stable; this can be seen as an Eckhaus instability. This steady state is stable in the range $8.13<\tilde{\mu}<8.36$, where the system exhibits the oscillatory instability described in Section 4.1, with $\tilde{d}_{-1}=0.71$, see Eq. (66) and Fig. 3d. The bifurcated solutions (Fig. 6a) are not reflection symmetric (one of the two possibilities anticipated at the end of Section 4.1). Now, the

$|-1|$

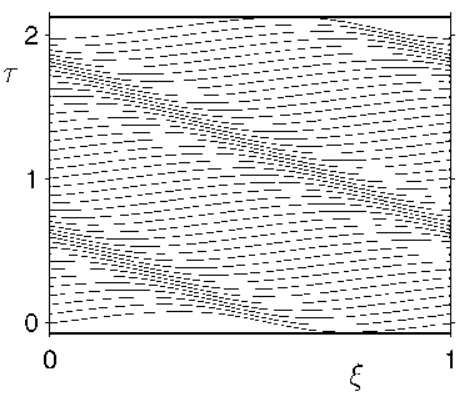

$|\cdot|$

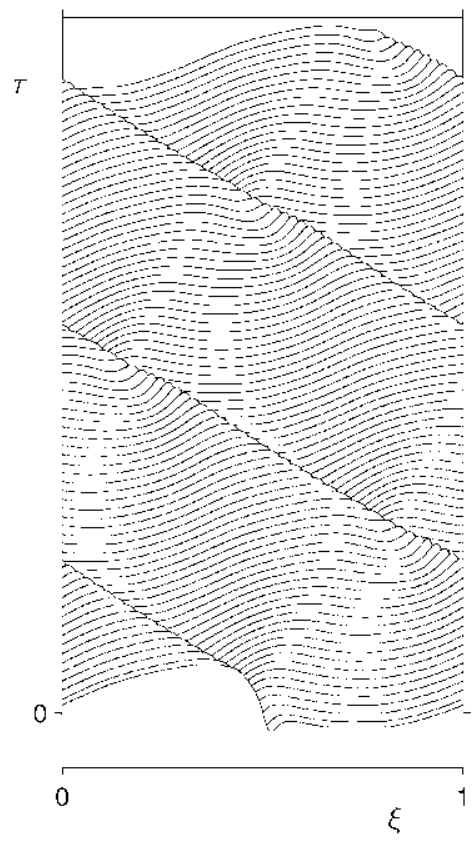

$|B|$

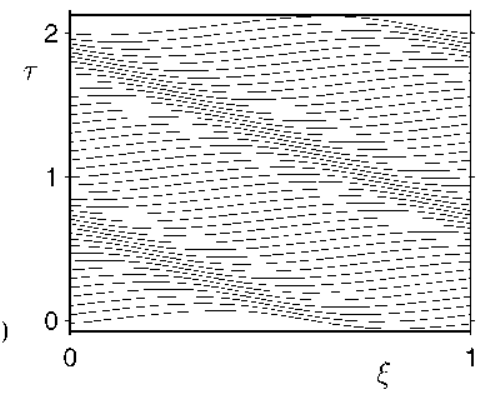

$|B|$

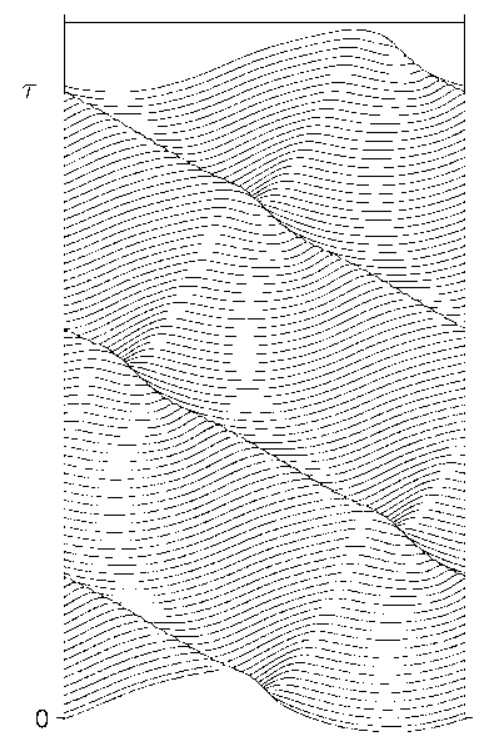

(b)

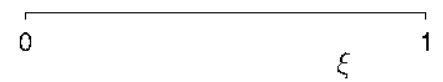

Fig. 6. Space-time plots of $|A|$ and $|B|$ for some attractors at $C_{g} L=1, \varepsilon=5 \times 10^{3}, S=0.05, \tilde{d}=-2.5$, and (a) $\vec{\mu}=8.36$ and (b) $\vec{\mu}=9.05$. 
whole pattern (in particular, both $A$ and $B$ ) travels with a constant speed 0.9 , which of course is not the group velocity, $A \simeq 0.51$. These solutions have been reported experimentally in [27] and could in principle appear as surprising if one expects that the envelopes $A$ and $B$ counterpropagate with the group velocity; this is necessarily true for patterns that exhibit small dispersive scales, but not for patterns that only exhibit the transport scale. In the range $8.36<\tilde{\mu}<9.10$ the system progressively exhibits a more complex spatial and temporal structure, yielding pulsating waves, in which the propagation velocity slightly oscillates, as in Fig. 6b. At $\tilde{\mu}=9.10$ the system suffers a new Eckhaus instability and jumps to the spatially uniform SW with wavenumber $m=-2$ (thus $\tilde{d}_{-2}=3.92$, see Eq. (66) and Fig. $3 \mathrm{~d}$ ), which loses stability at $\tilde{\mu}=9.11$, where it exhibits a standard oscillatory instability (like that in Section 4.1, case b); this yields again TWs for the wave envelopes, like that in Fig. 6a. In the range $9.11<\tilde{\mu}<11.21$, the system exhibits again various oscillatory instabilities, gains spatial structure, and loses temporal periodicity. At $\tilde{\mu}=11.21$, a new Eckhaus instability takes place, and the system jumps to a new propagating state that bifurcated (for a smaller value of $\tilde{\mu}$ ) from the branch of spatially uniform SWs with wavenumber $m=-3$ and $\tilde{d}_{-3}=7.13$. None of the patterns above is reflection symmetric. Thus, because of invariance under (62) the system also has in each case that pattern obtained form the one described above by reflection symmetry, which in particular travels in opposite direction.

Before proceeding further, two questions are relevant. First, the plots in Fig. 6a and b suggest that in the traveling patterns described above the dispersive scales are absent. This has been checked by repeating the calculations above for (a) decreasing values of the small parameter $\varepsilon$ and (b) for $\varepsilon=0$, obtaining in both cases the same transitions and attractors, albeit some small quantitative differences, at least for $8.36<\tilde{\mu}<10.01$. Secondly, these traveling patterns are born from spatially uniform SWs through oscillatory instabilities that would not be present if the mean flow were absent, as explained in Section 4.1. This has been checked by eliminating the integral term from Eqs. (51) and (52), and then integrating numerically the resulting equations with the same values of the coefficients. Now, the spatially uniform SW losses stability at $\tilde{\mu}=8.39$, where the system bifurcates supercritically to a nonsymmetric pair of dispersive wavetrains for the wave envelopes like that plotted in Fig. 7 (cf. Fig. 5a); this is just the second possibility anticipated at the end of Section 4.2 . This wavetrain remains stable up to $\tilde{\mu}=8.72$, where the system gains reflection symmetry and the solution becomes qualitatively similar to that in Fig. $5 \mathrm{a}$, which in turn suffers a oscillatory instability and yields a more complex dispersive pattern that remains stable up to $\mu=8.78$. Note that now, without mean flow, dispersive scales are not inhibited, which is in accordance with the results in [20].
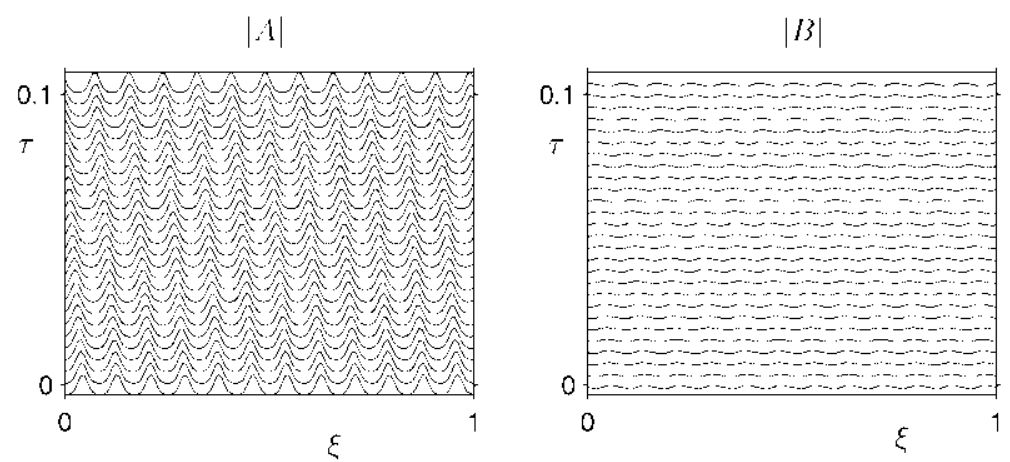

Fig. 7. Space-time plot of $|A|$ and $|B|$ for a attractor at $C_{g} L=1, \varepsilon=5 \times 10^{3}, S=0.05, \tilde{d}=-2.5, \vec{t}=8.5$, and no mean flow coupling. 
Case B At $S=0.5, \tilde{d}=0.5$ the spatially uniform SWs with $m=0$ exhibit a long wave oscillatory instability with wavenumber $n=1$ (1hus $\tilde{d}_{0}=0.5$, see Eq. (66) and Fig. 3a) at $\tilde{\mu}=9.55$, where the system bifurcales supercritically to a reflection symmetric pattern (the first possibility anticipated the end of Section 4.1 , case b), plotted for $\tilde{\mu}=9.56 \mathrm{in} \mathrm{Fig.} 8 \mathrm{a}$ (cr. Fig. 6a). These can be secn as beating states and are reminiscent of the oncs observed experimentally [27]. As $\tilde{\mu}$ increases, these altractors gain spatial structure but remain stable up to $\tilde{\mu}=10.3$, where the system exhibits a subcritical bifurcation and jumps to a new reflection symmetric spatially periodic state with wavenumber $n=2$, which in turn gains spatial struclure as $\tilde{\mu}$ increases; it first loses reflection symmety and then (at $\tilde{\mu}=11.2$ ) becomes temporally chaotic; an example of this chaotic attractor is given in Fig. 8b. Again, we have checked that dispersive scales are absent in all these solutions, at least for $\tilde{\mu}<11.8$.

Case C At $S=0.29, \tilde{d}=-1$, the spatially uniform SWs with $m=0$ remain stable for $\tilde{\mu}<9.4$, where they exhibit a long wave oscillatory instability (with $\tilde{d}_{0}=-1$, see Eq. (66) and Fig. 3b), and the system bifurcates supercritically to a branch of reflection symmetric patterns (the first possibility anticipated at the end of Section 2) with wavenumber $n=2$, like that plotted in Fig. 9a for $\tilde{\mu}=9.5$. These remain stable for $\tilde{\mu}<9.8$, where the system exhibits a subcritical Eckhaus instability and jumps to a new branch of reflection symmetric patterns (Fig. 9b), which in turn remain stable and reflection symmetric in the range $9.8<\tilde{\mu}<10.45$, albeit gaining some spatial structure and loosing temporal periodicity. At $\tilde{\mu}=10.45$ the pattern also lose reflection symmetry, which is not regained, at least for $\tilde{\mu}<14.75$, but remains otherwise qualitatively unchanged. Again, dispersive scales are absent in all solutions described above, at least for $\tilde{\mu}<11$.

Case D Finally, at $S=0.11, \tilde{d}=-0.5$, the primary instability of the spatially uniform SWs with $m=0$ occurs at $\bar{\mu}=8.17$ and is stationary (now $\tilde{d}_{0}=-0.5$, see Eq. (66) and Fig. 3c). But the bifurcated branch of reflection symmetric steady states (Fig. 10a) exhibits an oscillatory instability at $\bar{\mu}=8.26$, where the system bifurcates supercritically to abranch of reflection symmetric patterns similar to those in Case $B$ above (Fig. 8a), which subsequently gain spatio-temporal structure (Fig. 10b) but remain reflection symmetric and without dispersive scales, at least for $\bar{\mu}<9.49$.

Summarizing the results above, after destabilization of the spatially uniform steady states, the solutions of the system can cither be reflection symmetric or not, and either exhibil small dispersive scales or not. When gravity dominates, reflection symmetry is lost from the outset, and the system exhibits a pattern that travels undistorted to cither side (like some of the drifting patterns reported in [27]), depending on initial conditions. This is a source of drifting patterns that are fundamentally different from the ones encountered in [10], although both are essentially due to the presence of the viscous mean flow. Those in [10] exhibil a spatially uniform amplitude, the surface waves being thus reflection symmetric, and broken reflection sy mmetry (which is essential for the existence of drift [26]) manifests itself in the viscous mean flow, and not in the surface wave pattern (in first approximation). Here, instead, the drift of the paticrns is pushed by the broken rellection symmetry of the surface wave envelopes, whose spatial modulation is thus essential. Eckhaus instability (another global feature reported in [27]) is also frequent. The patterns exhibit temporal chaos as $\tilde{\mu}$ is increased. but not spatio-temporal chaos, which would require the presence of dispersive scales, as in [20], at least for the (realistic, but not too large) range of $\tilde{\mu}$ and the values of $k, S$, and $\tilde{d}$ checked above. Dispersive scales (which are paramount in the amplitude equations without mean flow [20]) seem to be inhibited by the viscous mean flow, even in cases when these are the most unstable scales in the primary instability of the spatially uniform steady states (Case A above). Thus, in most simulations above we could have neglected dispersion and consider the hyperbolic approximation of the amplitude Eqs. (51) and (52). But unfortunately, this cannot be done a priori because (a) dispersive scales could have been destabilized, as in [20], and (b) dispersive scales do appear for larger values of $\ddot{\mu}$ than those considered above, where complex dispersive 
1.1

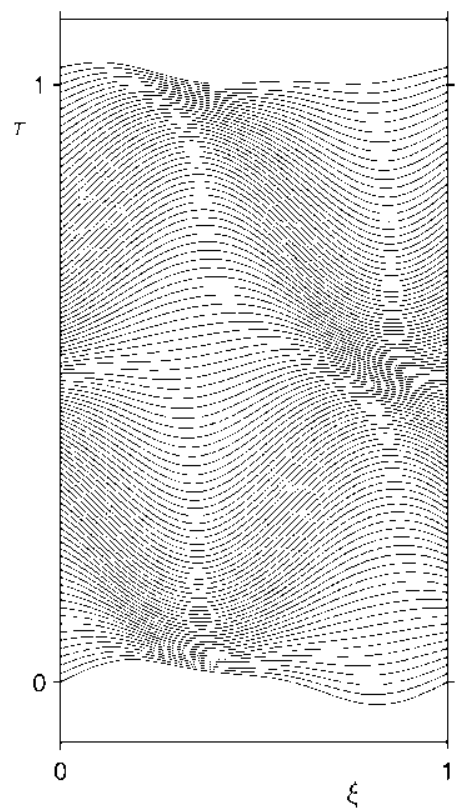

A

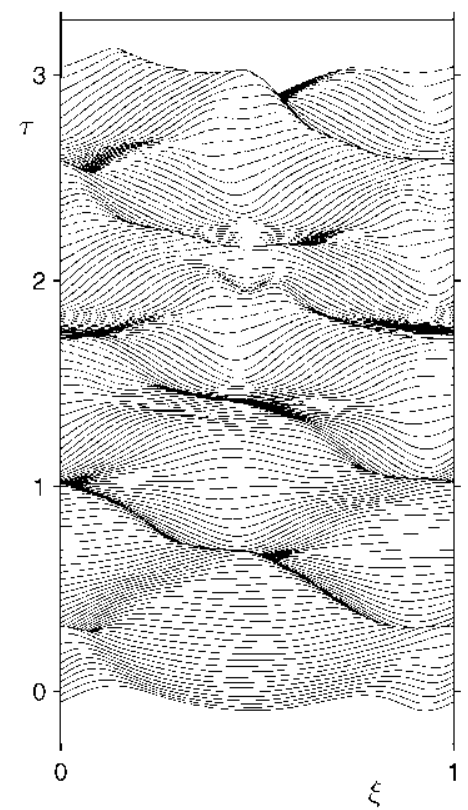

(a)

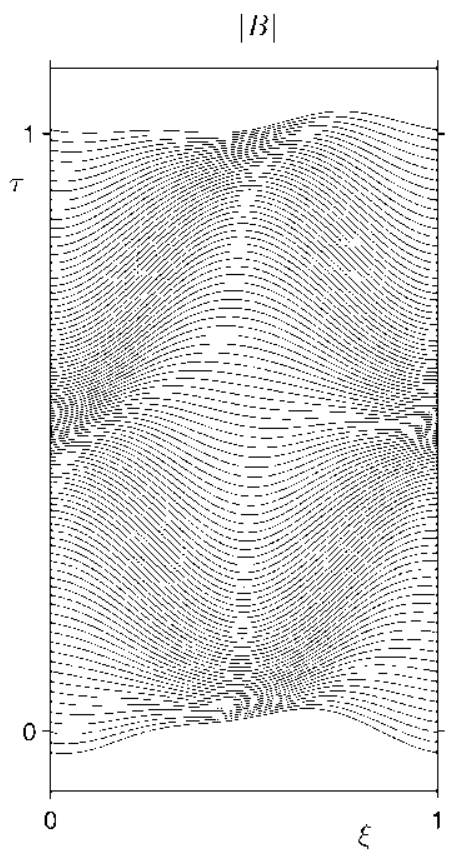

|B|

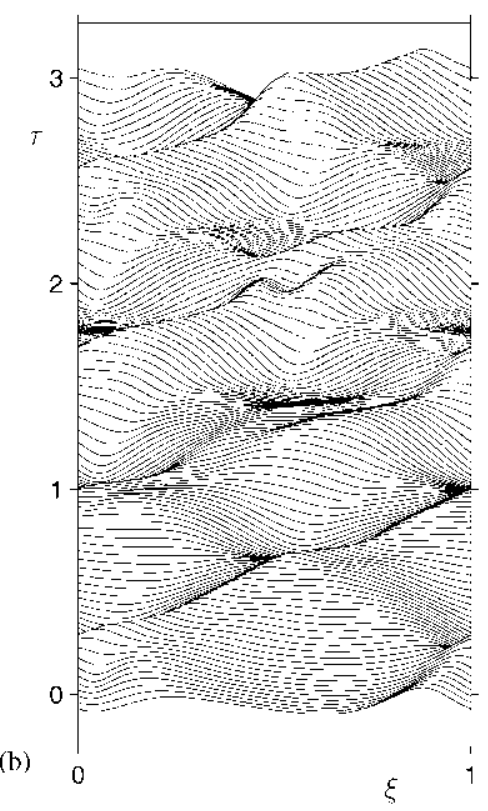

Fig. 8. Spacc-lime plots of $|A|$ and $|B|$ for some attractors at $C_{z} L=1, \varepsilon=5 \times 10^{-3}, S=0.5, \bar{d}=0.5$, and (a) $\bar{\mu}=9.56$ and (b) $\bar{\mu}=11.5$. 
$|A|$

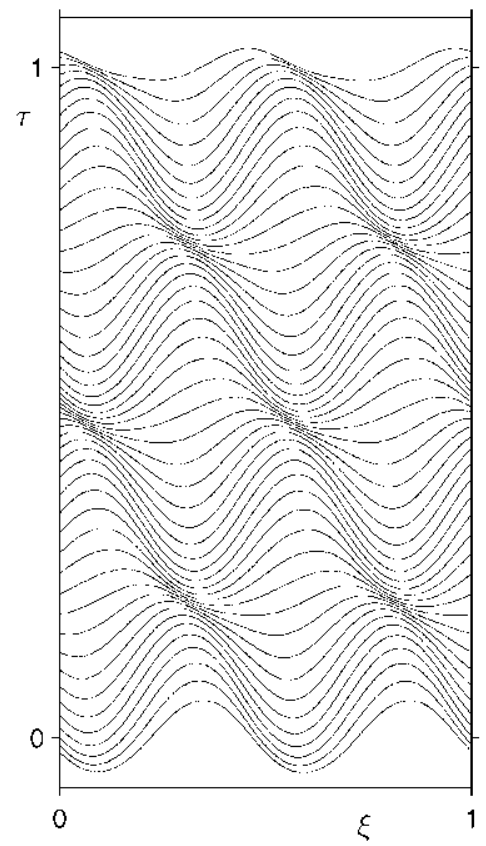

$A \mid$

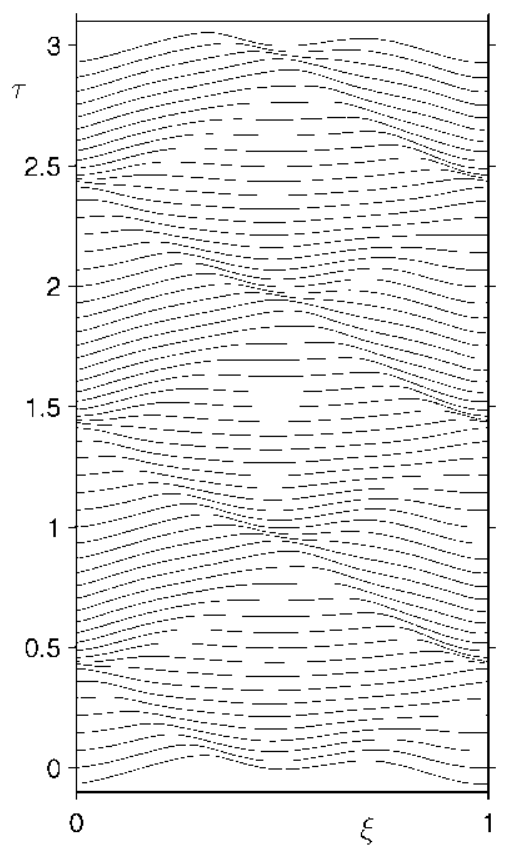

(a)

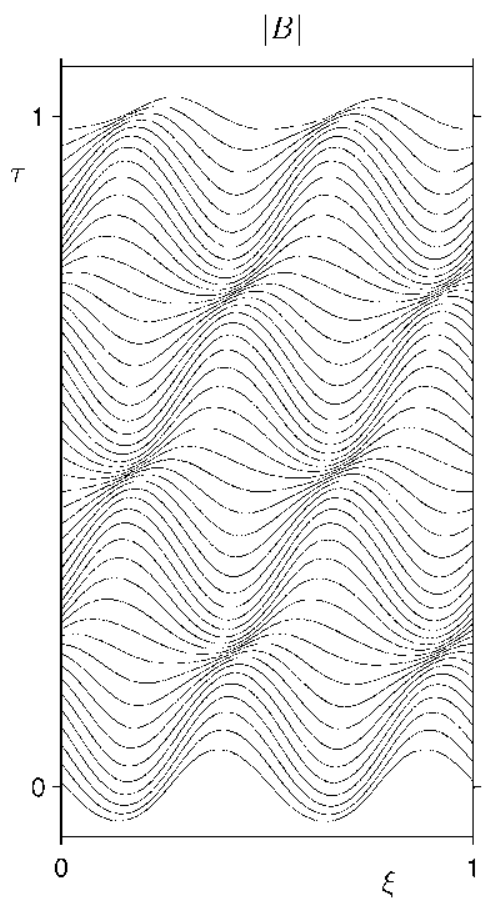

$|B|$

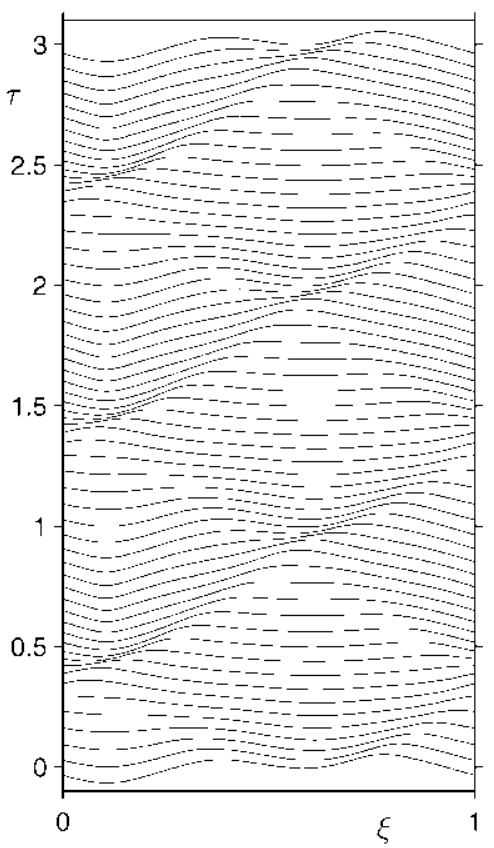

Fig. 9. Spacc-lime plots of $|A|$ and $|B|$ for some atractors at $C_{z} L=1, \varepsilon=5 \times 10^{-3}, S=0.29 . \tilde{d}=-1$, and (a) $\bar{\mu}=9.5$ and (b) $\bar{\mu}=9.9$. 
|A

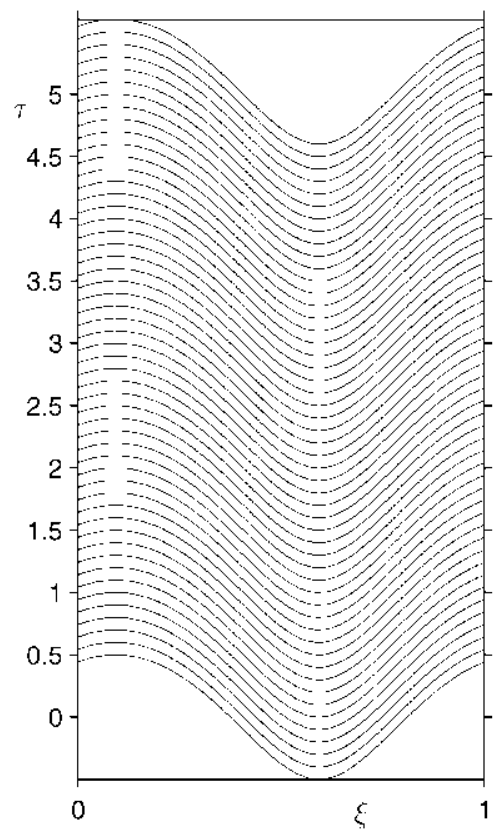

$|A|$

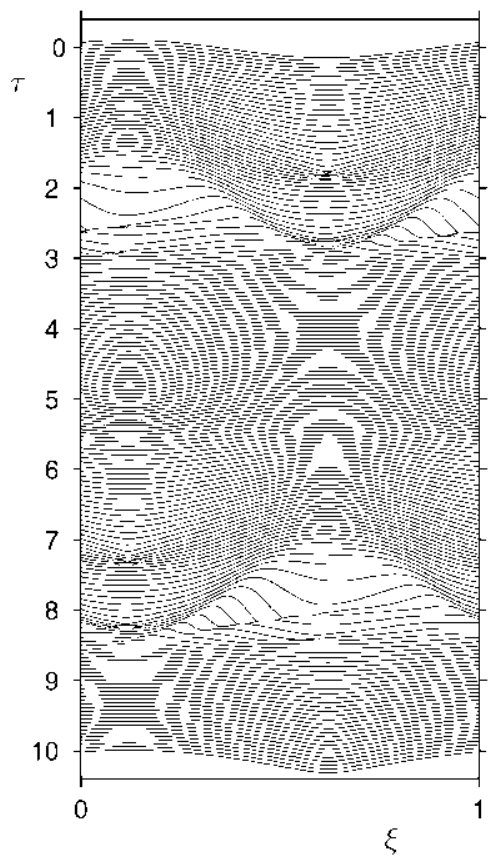

$|B|$

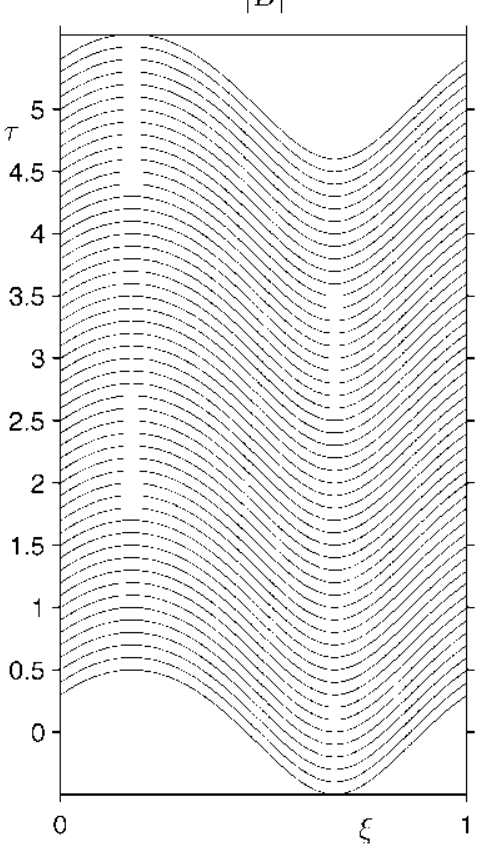

$|B|$

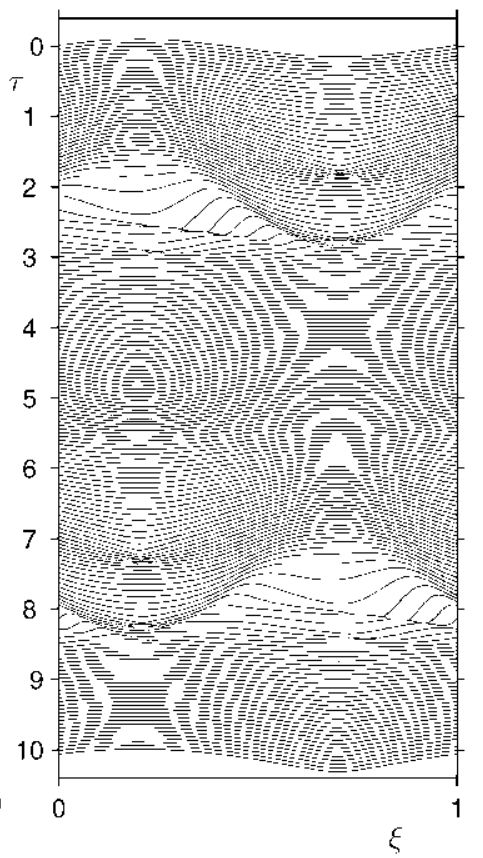

Fig. 10. Space-time plots of $|A|$ and $|B|$ for some attractors at $C_{1} L=1, \varepsilon=5 \times 10^{-3}, S=0.11, \tilde{d}=-0.5$, and (a) $\tilde{\mu}=8.2$ and (b) $\tilde{\mu}=8.71$. Note that time has been reversed in this plot. 
solution not presented here are obtained. The stabilizing effect of the mean flow is far from evident, even though it exhibits viscous effects. Note that viscous diffusion is absent in the horizontal direction, in which dispersive scales cxhibit fast oscillations.

\subsection{Mean flow patterns}

Let us now turn to the structure of the mean flow associated with some of the attractors described above, namely the simplest ones. The time dependent mean flow patterns associated with more complex attractors exhibit a lot of unsteady structure, whose description and interpretation is subule, and outside the scope of this paper.

In order to somewhat visualize patterns we shall plot color maps of the vorticity associated with the mass transport velocity (61), namely

$$
\Omega^{\mathrm{mt}}=\varphi_{y y}^{m}+8 \omega k^{2} \mathrm{e}^{2 k y}\left(|A|^{2}-|B|^{2}\right) .
$$

Note that the forcing shear stress in the second condition of (54) and the Stokes drift are both proportional to $|A|^{2}-|B|^{2}$. Thus we must expect that the flow exhibits counter-rotating eddies below those regions of the free surface where $|A|>|B|$ and $|A|<|B|$. But for unsteady patterns the interpretation of vorticity contours requires some care. Because of unsteady effects, neither the strength of each eddy needs to be as suggested by the vorticity values, nor its size needs to correspond, even approximately with the region where the vorticity exhibits a constant sign.

The mass transport vorticity below the surface waves in Fig. 5a is plotted in Fig. 11, and is periodic in time; in fact, only a half of the period is considered in Fig. 11, the pattern in the second half being obtained from the plotted one by reflection symmetry. The period and horizontal wavenumber are much shorter than their counterparts in the remaining periodic patterns below, which only exhibit the much larger transport scales. The pattern consists of an array of counter-rotating eddies (in that part of the period such that $|\Lambda|^{2}-|B|^{2}$ alternatively exhibits opposite signs as $\xi$ increases), which subsequently merge (when $|A|^{2}-|B|^{2}$ vanishes), and rotate in opposite direction.

The mass transport vorticity associated with the TW in Fig. Ga travels undistorted to the left (at a velocity $c \simeq 0.9$ ) with the primary pattern, and is plotted in Fig. 12. According to the scaling (50) and (60), in the original variables used in (1)-(4) the propagation velocity of the pattern $\left(c /\left(C_{g} L\right) \sim 1\right)$ is much larger than the local fluid velocity $\left(\psi_{y}^{m} \sim C_{g} \ll 1\right)$. Thus we do not plot the streamlines in a reference frame moving with the pattern, which are horizontal in first approximation.

The mass transport vorticity below the reflection symmetric pattern in Fig. 8a is plotted in Fig. 13. Note that these are somewhat similar to those in Fig. 11, the main difference being that the spatial wavelength is much larger now.

The mass transport vorticity produced by reflection symmetric pattern in Fig. 9a is similar to that in Fig. 13, except for the fact that the spatial period of the mean flow pattern is now one-half of that in Fig. 13.

Finally, the steady mass transport vorticity and streamlines associated with the pattern in Fig. 10a are plotted in Fig. 14a and $b$.

In the remaining of this section we briefly explain the numerical method used to integrate Eqs. (51)-(57). The solution is first expanded in Fourier series in $\xi$

$$
\begin{aligned}
& \left(\begin{array}{l}
A(\xi, t) \\
B(\xi, t)
\end{array}\right)=\sum_{n=-\infty}^{\infty}\left(\begin{array}{c}
A_{n}(t) \\
B_{n}(t)
\end{array}\right) \mathrm{e}^{\mathrm{i} 2 \pi n \xi}, \\
& \left(\begin{array}{c}
F(\xi, t) \\
\varphi(\xi, y, t)
\end{array}\right)=\sum_{n=-\infty}^{\infty}\left(\begin{array}{c}
F_{n}(t) \\
\psi_{n}(y, t)
\end{array}\right) \mathrm{e}^{\mathrm{i} 2 \pi n \xi}, \quad\left(\begin{array}{l}
F_{n} \\
\varphi_{n}
\end{array}\right)=\left(\begin{array}{c}
\bar{F}_{-n} \\
\bar{\varphi}_{-n}
\end{array}\right) .
\end{aligned}
$$



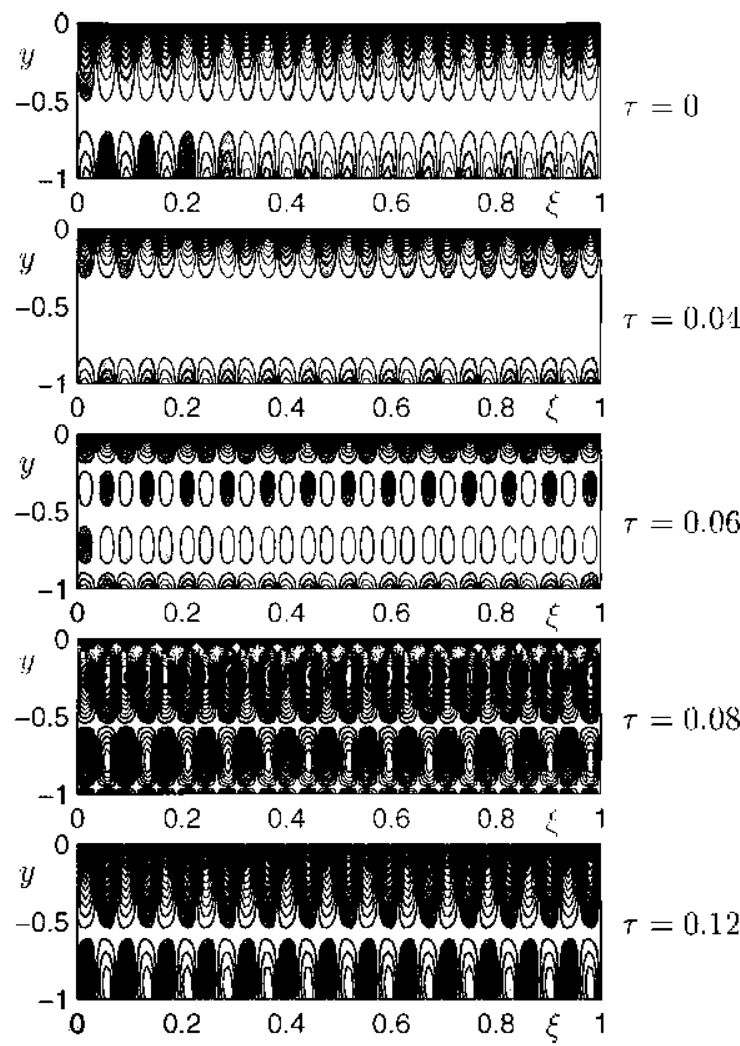

Fig. 11. Mass transport vorticity color (red positive vorticity, blue negative vorticity) maps produced by the periodic surface wave pattem in Fig. 5 a.

The resulting equations for the $n$th mode of the mean flow are

$$
\begin{aligned}
& \varphi_{n_{y y t}}=\varphi_{n_{y y g y}} \text { in }-1<y<0, \\
& (2 \pi n) \varphi_{n}-F_{n_{\bar{r}}}=2 \omega(2 \pi n) \Gamma|B|^{2}-|A|^{2} l_{n}, \quad \varphi_{n_{y y}}=8 \omega k^{2}\left[|\Lambda|^{2}-|B|^{2} l_{n},\right. \\
& \varphi_{n_{y y}}-\varphi_{n_{y \tau}}+\Gamma(2 \pi n) F_{n}=0 \text { at } y=0,
\end{aligned}
$$

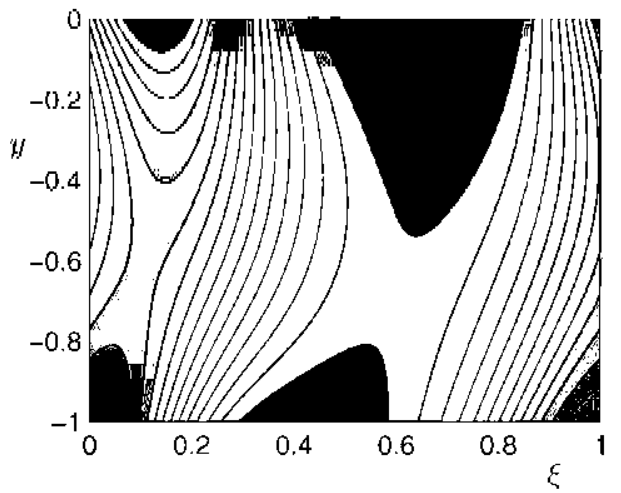

Fig. 12. Mass transport vorticily color map produced by the surface wave pattem in Fig. $6 a$. 

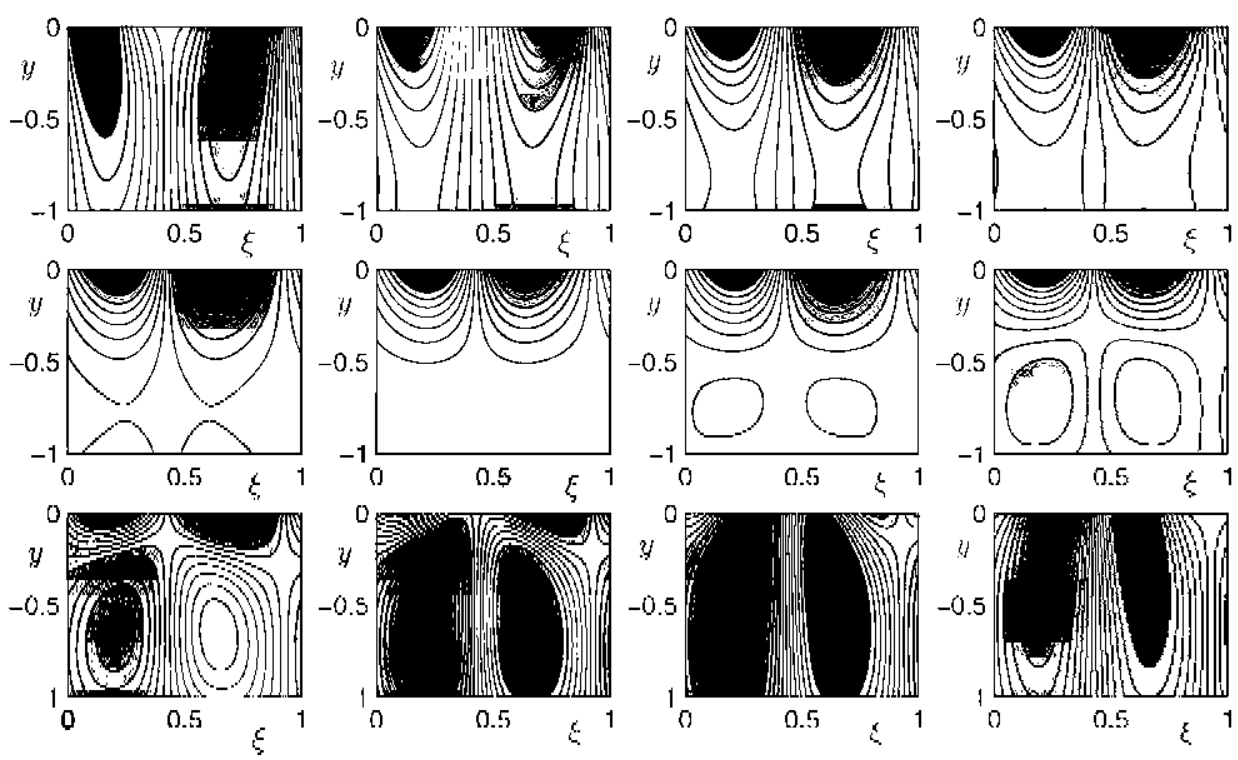

Fig. 13. Mass transport vorticity color maps produced by the periodic surface wave pattern in Fig. 8a.

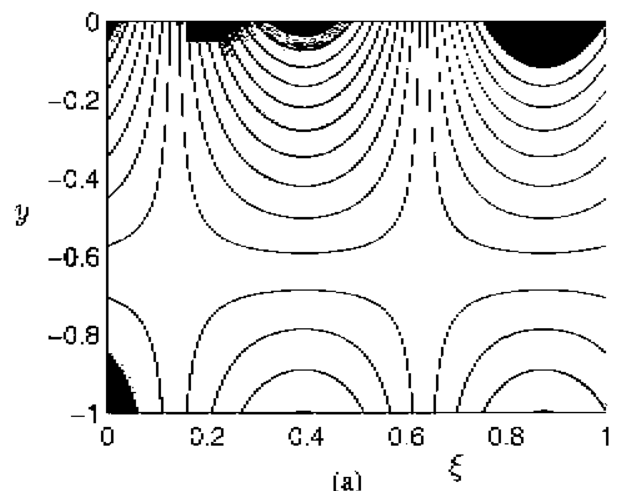

(a)

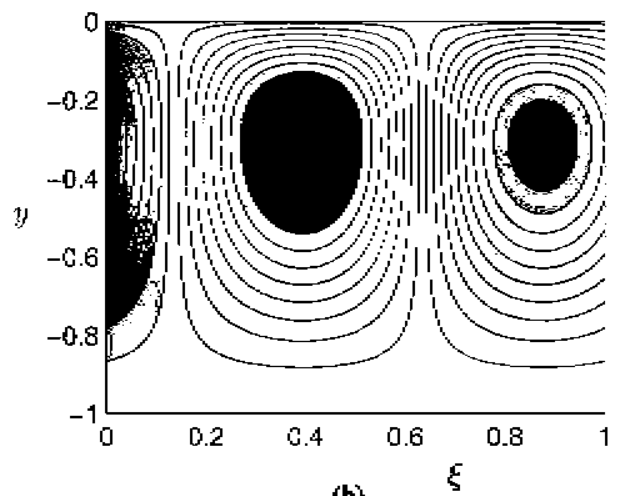

(b)

Fig. 14. Mass transport vorticity color maps (a) and streamlines (b) produced by the steady surface wave pattern in Fig. 10a. 


$$
\begin{aligned}
& \varphi_{0 y y}=\varphi_{n}=\varphi_{n_{y}}=0 \text { at } y=-1, \\
& F_{0}=0 .
\end{aligned}
$$

This is a linear nonhomogeneous problem and its general solution can be expressed as

$$
\left(\begin{array}{c}
F_{n}(t) \\
\varphi_{n}(y, t)
\end{array}\right)=\sum_{j=0}^{\infty} c_{n j}(t)\left(\begin{array}{c}
F_{n j} \\
\varphi_{n j}(y)
\end{array}\right)+p_{n}(t)\left(\begin{array}{c}
F_{p n} \\
\varphi_{p m}(y)
\end{array}\right)
$$

where $\left(F_{n j}, \varphi_{n j}\right)$ are the eigenfunctions of the homogeneous (i.e., $\left[|\Lambda|^{2}-|B|^{2} l_{n}=0\right.$ ) version of (100)-(103), $\left(F_{p n}, \varphi_{p n}\right)$ is the steady particular solution of the system $(100)-(103)$ with the right-hand side of the first condition of Eq. (101) set to 0, and the right-hand side of the second condition of Eq. (101) set to 1, and $p_{n}(t)=\left.8 \omega k^{2}|| \Lambda\right|^{2}-$ $|B|^{2} l_{n}$.

Now, by inserting the expansions (104) into (100)-(103), and inserting (98), (99) and (104) into (51)-(57), an infinite system of ODEs is obtained for the evolution of the coefficients $\Lambda_{n}(t), B_{n}(t)$ and $c_{n j}(t)$. This system is truncated $\left(n_{\max }=256\right.$ and $j_{\max }=32$, typically) and numerically integrated using a fourth-order Runge-Kutta method with time step $\Delta t=0.001$.

\section{Concluding remarks}

We have derived a system of CAMF equations for the evolution of two counterpropagating wavetrains (with opposite phase velocity) and the associated viscous mean flow. The starting point was a more general system of coupled equations derived in [8]. The main simplification is seen in the mean flow equations, which are linear here because the mean flow is almost parallel.

In order to illustrate the feasibility of the theory described here, we describe an experimental realization. As in [27], we consider an annular container with a $110 \mathrm{~mm}$ diameter and $4 \mathrm{~mm}$ depth (a half of that in [27], to obtain an appropriately large aspect ratio), filled with extremely clean water (see [28]) and vertically vibrated with a $36.6 \mathrm{~Hz}$. Using $T=72 \mathrm{dyn} / \mathrm{cm}$, the capillary-gravity time is $0.017 \mathrm{~s}$ and hence $\omega=1.93$. The remaining nondimensional parameters are $L=86.4, S=0.31, C_{g}=1.04 \times 10^{-3}, k=2, \delta=0.011, d=-0.04$ and $v_{\mathrm{g}}=1.17$. Note in particular that $v_{g} / L=0.014 \sim \delta$, and that the remaining assumptions (36) hold.

The simplest nonflat solutions are (as always in the Faraday system) spatially uniform SWs, whose linear stability has been analyzed in Section 4. Two essentially different instabilities have been identified. Namely, (i) long wave instabilities that only exhibit transport scales and yield either stationary waves or oscillatory waves; the latter can in turn either be reflection symmetric or not, and would be absent if the effect of the mean flow on the surface waves were ignored; and (ii) short wave, dispersive instabilities that are convected at the group velocity.

For still larger values of the forcing frequency we have integrated the CAMF equations to obtain several representative large-time attractors. The simplest ones are (a) dispersive wavetrains (Fig. 5a) and (b) patterns that only cxhibit the transport scales, which for the wave envelopes are either (b-1) time-periodic and reflection symmetric (Fig. 8a), (b-2) nonreflection symmetric, which travel undistorted at a constant specd (Fig. 6a), or (b-3) stationary and nonuniform (Fig. 10a). The system also cxhibits more complex, chaotic patterns for larger forcing amplitudes, but these have not been analyzed. Instead we have concentrated in those simpler patterns that could be casily identified in experiments, in an attempt to stimulate experimental visualizations and measurements, with special attention on the associated mean flow, which has not been paid so far. 


\section{Acknowledgements}

This work was supported by the Spanish Dirección General de Investigación under Grant BFM200)1-2363 and by the National Acronautics and Space Administration under Grant NAG3-2152.

\section{References}

[1] M. Faraday, On the forms and states assumed by fluids in contact with vibrating elastic surfaces, Phil. Trans. Roy. Soc. London 121 (1831) 319-340.

[2] J.W.S. [ ord Rayleigh, On the crispations of fluid resting upon a vibrating support, Phil. Mag. 16 (1883) $50-58$.

[3] J. Miles, D. IIenderson, Paranetrically forced surface waves, Annu. Rev. Fluid Mech. 22 (1990) 143-165.

[4] M.C. Cross, P.C. IIohenberg, Patten formation oulside of equilibrium, Rev. Modern Phys. 65 (1993) 851-1112.

[5] S. Fauve, Parametric instabilities, in: G. Martinez Mekler, T.H. Seligman (Eds.), Dynamics of Nonlinear and Disordered Systems, World Scientific, Singapore, 1995 , pp. 67115.

[6] A. Kudrolli, J.P. (iollub, Patterns and spatio-temporal chaos in parametrically foreed surfaes waves: a systematic survey at large aspeet ratio, Physica D $97(1997) 13.3-154$.

[7] J.A. Nicolás, J.M. Vega, Weakly nonlinear oscillations of axisymmetric liquid bridges, J. Fluid Mech. 328 (1996) 95-128.

[87 J.M. Vega, E. Knobloch, C. Martel, Nearly inviscid Faraday waves in annular containers of moderately large aspect ratio, Physica D 154 (2001) 313336

[9] M.J. Higuera, J.M. Vega, E. Knobloch, Interaction of nearly inviscid, multi-mode Faraday waves and mean flows, in: L.L. Bonilla, G. Platero, D. Reguera, J.M. Rubi (F,ds.), Coherent Structures in Complex Systems, Springer, Berlin, 2001, pp. $328-337$.

[10] F.. Martin, C. Martel, J.M. Vega, Drift instability of standing Faraday waves in an annular container, J. Fluid Mech. 467 (2002) 57-79.

[11] E. Knobloch, J.M. Vega, Nearly inviscid Faraday waves, in: P. Newton, P. IIolmes, A. Weinstein (Eds.), Geometry, Mechanics and Dynanics: Volume in IIonor of the 60th Birthday of J.D. Marsden, Springer, Berlin, 2002, pp. 181-222.

[12] M.C.W. Jones, Nonlinear stability of resonant capillary-gravity waves, Wave Motion 15 (1992) 267283.

[13] P. Christodoulides, F. Dias, Resonant gravity-capillary interfacial waves, J. Fluid Mech. 265 (1994) 303343.

[14] I.F. Mccioldrick, On Wilton ripples: a special case of resonant interactions, J. Fluid Mech. 42 (1970) 193-200).

[15] K. Trulsen, CC. Mei, Modulation of three resonating gravity-capillary waves by a long gravity wave, J. Fluid Mech. 290 (1995) 345-376.

[16] K. Trulsen, C.C. Mei, Effect of weak wind and dimping on Wilton's ripples, J. Fluid Mech. 335 (1997) 141-163.

[17] C. Marlel, E. Knobloch, Damping of nearly inviscid water waves, Phys. Rev. E 56 (1997) 5544-5548.

[18] J.V. Wehausen, E.V. Laitone, Surface waves, in: S. Fleige (Ed.), Encyclopedia of Physics, vol. 9, Fluid Dynamics III (Handbuck der Physik), Springer, Berlin, 1960.

[19] C. Martel, F. Knobloch, J.M. Vega, 1)ynamics of counterpropagating waves in parametrically foreed systems, Physica 1) 137 (20)(0) 94-123.

[20] C. Martel, J.M. Vega, F. Knobloch, Jynamics of eounterpropagating waves in parametrically driven systems: dispersion vs. advection, Physica D 137 (2002) 94-123.

[21] M.S. Longuet-Higgins, Mass transport in water waves, Phil. Trans. Roy. Soc. A 245 (1953) $535-581$.

[22] G.K. Batchelor, An Introduction to Fluid Dynamics, Cambridge University Press, Cambridge, 1967.

[23] M. Umeki, Lagrangian motion of fluid particles induced by three-dimensional standing surface waves, Plyys. Fluids $\Lambda 4$ (1992) 19681978.

[24] \%.C. Feng, S. Wiggins, Fluid particle dynamies and Stokes drift in gravity and eapillary waves generated by the Faraday instability, Nonlinear Dyn. $8(1995)$ 141-160.

[25] A. Davey, S. Stewartson, On three-dimensional packets of surface waves, Proc. Roy. Soc. London A 338 (1974) 101-1 10.

[26] J.D. Crawford, E. Knobloch, Symmetry and symmetry-breaking bifurcations in fluid mechanics, Ann. Rev. Fluid Mech. 23 (1991) $341-387$.

[27] S. Douady, S. Fauve, O. Thual, Oscillatory phase modulation of parametrically forced surface waves, Europhys. Lett. 10 (1989) 309315.

[28] D.M. Henderson, J.W. Miles, Surface-wave damping in a circular cylinder with a fixed contact line, J. Fluid Mech. 275 (1994) 285299. 